\title{
Targeting CD137 enhances the efficacy of cetuximab
}

\author{
Holbrook E. Kohrt, ${ }^{1}$ A. Dimitrios Colevas, ${ }^{1}$ Roch Houot, ${ }^{1,2,3}$ Kipp Weiskopf, ${ }^{1}$ \\ Matthew J. Goldstein, ${ }^{1}$ Peder Lund, ${ }^{1}$ Antonia Mueller, ${ }^{1}$ Idit Sagiv-Barfi, ${ }^{1}$ \\ Aurelien Marabelle, ${ }^{1,4}$ Ruth Lira, ${ }^{1}$ Emily Troutner, ${ }^{1}$ Lori Richards, ${ }^{1}$ Amanda Rajapaska, ${ }^{1}$ \\ Jonathan Hebb, ${ }^{1}$ Cariad Chester, ${ }^{1}$ Erin Waller, ${ }^{1}$ Anton Ostashko, ${ }^{1}$ Wen-Kai Weng, ${ }^{1}$ \\ Lieping Chen, ${ }^{5}$ Debra Czerwinski, ${ }^{1}$ Yang-Xin Fu, ${ }^{6}$ John Sunwoo, ${ }^{1}$ and Ronald Levy ${ }^{1}$
}

\begin{abstract}
'Department of Medicine, Division of Oncology, Stanford University, Stanford, California, USA. ${ }^{2} \mathrm{CHU}$ Rennes, Service Hématologie Clinique, Rennes, France. 3INSERM, U917, Rennes, France. ${ }^{4}$ Centre de Recherche en Cancérologie de Lyon, UMR INSERM U1052, CNRS 5268, Centre Léon Bérard, Université de Lyon, Lyon, France. ${ }^{5}$ Department of Immunobiology, Yale Cancer Center, New Haven, Connecticut, USA. ${ }^{6}$ Department of Pathology and Committee on Immunology, University of Chicago, Chicago, Illinois, USA.
\end{abstract}

\begin{abstract}
Treatment with cetuximab, an EGFR-targeting IgG1 mAb, results in beneficial, yet limited, clinical improvement for patients with head and neck (HN) cancer as well as colorectal cancer (CRC) patients with WT KRAS tumors. Antibody-dependent cell-mediated cytotoxicity (ADCC) by NK cells contributes to the efficacy of cetuximab. The costimulatory molecule CD137 (4-1BB) is expressed following NK and memory T cell activation. We found that isolated human NK cells substantially increased expression of CD137 when exposed to cetuximab-coated, EGFR-expressing HN and CRC cell lines. Furthermore, activation of CD137 with an agonistic $\mathrm{mAb}$ enhanced NK cell degranulation and cytotoxicity. In multiple murine xenograft models, including EGFR-expressing cancer cells, HN cells, and $K R A S$-WT and $K R A S$-mutant CRC, combined cetuximab and anti-CD137 mAb administration was synergistic and led to complete tumor resolution and prolonged survival, which was dependent on the presence of NK cells. In patients receiving cetuximab, the level of CD137 on circulating and intratumoral NK cells was dependent on postcetuximab time and host FcyRIIIa polymorphism. Interestingly, the increase in CD137-expressing NK cells directly correlated to an increase in EGFR-specific $\mathrm{CD8}^{+} \mathrm{T}$ cells. These results support development of a sequential antibody approach against EGFR-expressing malignancies that first targets the tumor and then the host immune system.
\end{abstract}

\section{Introduction}

Colorectal carcinoma (CRC) is estimated to remain the third most common cancer, affecting over 140,000 lives and leading to over 50,000 deaths in the United States in 2013 alone. Though cases of head and neck $(\mathrm{HN})$ cancer are 3 -fold less common, the incidence continues to rise due to a growing population infected with human papillomavirus (HPV) (1). EGFR ERBB1 (avian erythroblastic leukemia viral [v-erb-b] oncogene homolog, receptor for EGF) is a poor prognostic marker in multiple solid tumors, including CRC, HN, non-small cell lung, and pancreatic carcinomas, and is a therapeutic target of mAbs (cetuximab and panitumumab) as well as small molecule kinase inhibitors (erlotinib and gefitinib). Cetuximab, a human-mouse chimeric IgG1 mAb, was approved by the Food and Drug Administration first in 2004 and today is indicated for patients with CRC and HN carcinomas (2). However, responses to cetuximab monotherapy are limited; only 1 in 5 patients with metastatic CRC (3) and only 1 in 10 with advanced HN carcinoma respond to treatment $(4,5)$. Despite expression of the target, patients with non-small cell lung, pancreatic, and CRC with codon 11 or 12 KRAS mutations fail to benefit from cetuximab (6).

Given multiple mechanisms of cetuximab's action, a variety of approaches have been pursued to enhance its efficacy. By competitively blocking the binding of EGF, phosphorylation and kinase activation are prevented, thereby inhibiting cell growth, inducing apoptosis, and decreasing production of matrix metalloproteinase and vascular endothelial growth factor $(7,8)$. In addition to these

Conflict of interest: The authors have declared that no conflict of interest exists. Citation for this article: J Clin Invest. 2014;124(6):2668-2682. doi:10.1172/JCI73014. in vitro effects, in vivo evidence in both murine models and patients suggests cetuximab's efficacy is due to antibody-dependent cellmediated cytotoxicity (ADCC), which requires immune effector cells, mainly NK cells, binding via their Fc receptor (Fc $\gamma$ RIII, CD16) to the IgG1 Fc, heavy-chain, portion of cetuximab (9-13). Targeting EGFR by small molecules that lack an Fc, and therefore lack ADCC, has not resulted in a clinical benefit in HN or CRCs. Supporting ADCC as a primary mechanism of cetuximab's activity in patients, NK cell infiltrate within primary colorectal tumors independently predicts prognosis (14). Patients with colorectal and HN carcinomas harboring a high-affinity Fc $\gamma$ RIII polymorphism have been shown to respond more favorably to cetuximab both ex vivo with higher cytotoxicity against EGFR-expressing cell lines (15) and clinically with superior disease-free and overall survival (15-19). Therefore, methods to enhance ADCC, such as stimulating the innate immune response, may clinically translate to improved antitumor activity. Augmenting the NK cell response to cetuximab therapy may enhance the adaptive immune response in addition to innate immunity due to NK cell-DC "crosstalk," which leads to tumor antigen-specific T cell responses following cetuximab therapy (20).

We sought to identify an inducible and targetable costimulatory molecule on NK cells in order to enhance ADCC. CD137 (4-1BB) is upregulated on human NK cells when they encounter antibody-bound tumor cells (21). Therefore, we hypothesized that the antitumor efficacy of cetuximab could be improved through a dual antibody strategy: first by inducing CD137 expression on NK cells upon their exposure to cetuximab-bound tumor cells and subsequently by targeting activated NK cells with an agonistic anti-CD137 $\mathrm{mAb}$. 

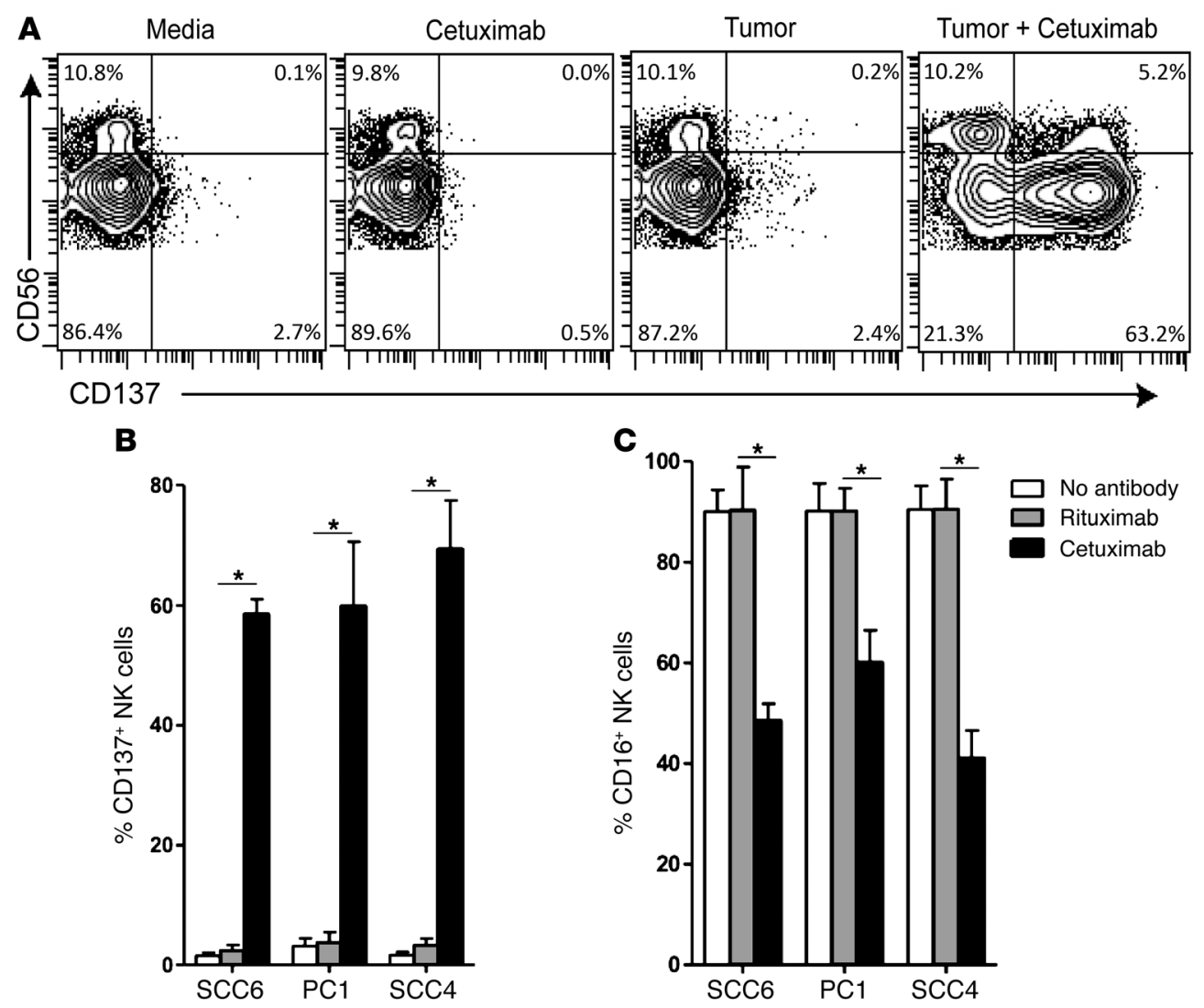

Figure 1

Cetuximab induces CD137 upregulation on human NK cells following incubation with EGFR-positive tumor cells. Peripheral blood from healthy donors was analyzed for CD137 expression on CD3-CD56+ NK cells after 24-hour culture with EGFR-positive tumor cell lines SCC6, PC1, and SCC4, and cetuximab or rituximab. (A) Percentage of NK cells divided by quadrant to delineate subsets of CD3-CD56 bright and CD3-CD56 dim expressing CD137 from a representative healthy donor after 24-hour culture with the EGFR-positive tumor cell line PC1 and cetuximab. (B) Percentage of CD137 expression on NK cells from 3 healthy donors after 24-hour culture with the EGFR-positive tumor cell line SCC6, PC1, or SCC4, and cetuximab or rituximab. (C) CD16 expression on NK cells from a 3 healthy donors after 24-hour culture with the EGFR-positive tumor cell line SCC6, PC1, or SCC4, and cetuximab or rituximab. ${ }^{*} P<0.001$.

\section{Results}

Cetuximab induces CD137 upregulation on buman NK cells following incubation with EGFR-positive tumor cells. CD137 expression was induced on the surface of NK cells from healthy human subjects following incubation with cetuximab and EGFR-expressing cancer cell lines (SCC6, PC1, and SCC4) (Figure 1A). This CD137 upregulation required the presence of both an EGFR-expressing cell and an EGFR-targeting mAb, as little effect on CD137 expression was observed with cetuximab or with EGFR-expressing cancer cell lines alone. Similarly, NK cell expression of CD137 did not increase following culture with a non-EGFR-targeting $\mathrm{mAb}$, rituximab, which targets CD20, even in the presence of the EGFRexpressing cells (Figure 1, B and C). The induction of CD137 occurred preferentially in CD56 $6^{\mathrm{dim}}$ compared with CD56 $6^{\text {hi }} \mathrm{NK}$ cells and among this subset was associated with a concurrent decrease in the expression of the Fc $\gamma$ RIII (CD16) (Figure 1, A-C).

Anti-CD137 agonistic mAb increases cetuximab-mediated NK cell cytotoxicity on tumor cells and DC cytokine secretion. To determine whether CD137 is a potential therapeutic target for enhancing NK cell function, NK cells were first activated to express CD137 by their exposure to EGFR-expressing cancer cells and cetuximab. Activated CD137-expressing NK cells were then reisolated and tested for their ability to perform ADCC against EGFR-expressing cancer cells (Figure 2, A-F). Activated NK cells showed enhanced ADCC following anti-CD137 mAb stimulation, as measured by apoptosis (Figure 2, $\mathrm{A}-\mathrm{C}$ ) and chromium release (Figure 2, D-F) from EGFR-expressing cancer cells. Though the anti-CD137 mAb enhanced cytotoxicity, specifically ADCC, enhanced IFN- $\gamma$ secretion, another usual measure of NK cell function, in contrast, was not observed (Supplemental Figure 1, A-C; supplemental material available online with this article; doi:10.1172/JCI73014DS1). However, in the presence of activated NK cells, EGFR-expressing cancer cells, cetuximab, and immature DCs (iDCs), anti-CD137 agonistic mAb enhanced secretion of IL-12, IFN- $\gamma$, and TNF- $\alpha$ (Supplemental Figure 1, D-F).

Anti-CD137 agonistic mAb enhances antitumor activity of cetuximab in vivo against both KRAS-mutant and KRAS-WT tumors. We tested the ability of cetuximab to induce CD137 expression on murine NK cells in vivo in an EGFR-expressing xenotransplant model (SCC6). We administered cetuximab 21 days following implantation of tumor and measured the expression of CD137 on NK cells in the peripheral blood, spleen, and tumor (Figure $3 \mathrm{~A}$ ). In each of the 3 compartments and at several time points, we observed upregulated expression of CD137 on NK cells (Figure 3, B and C). Interestingly, at the tumor site, CD137 expression peaked at 24 hours following 
A
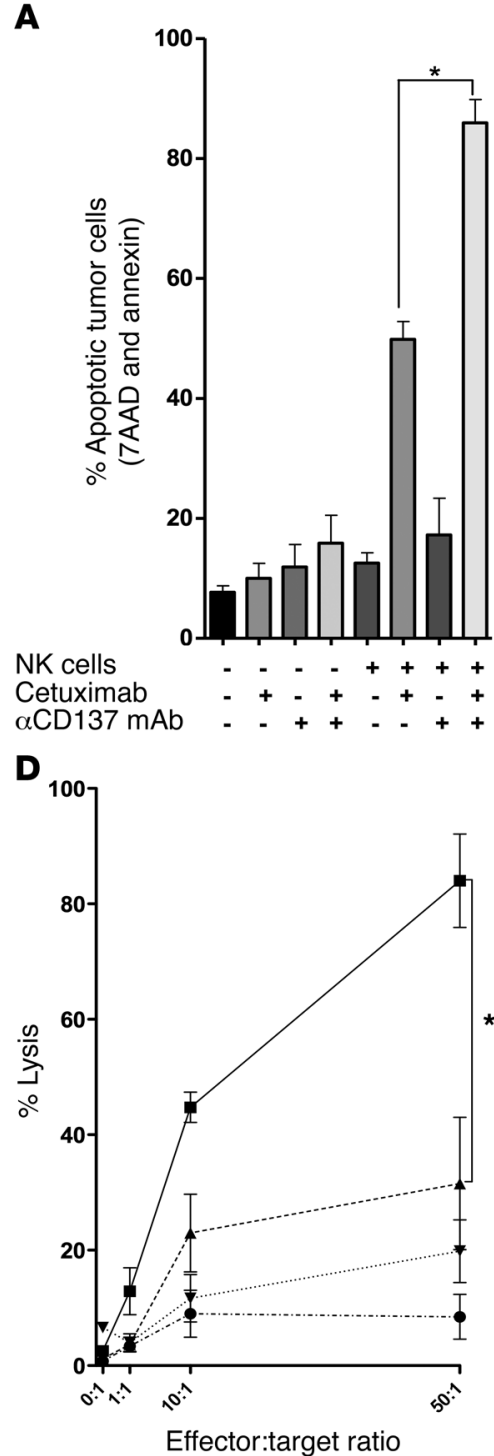

B

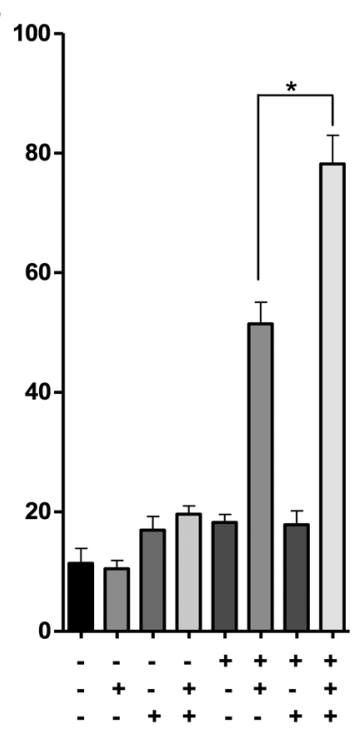

$E_{100}$

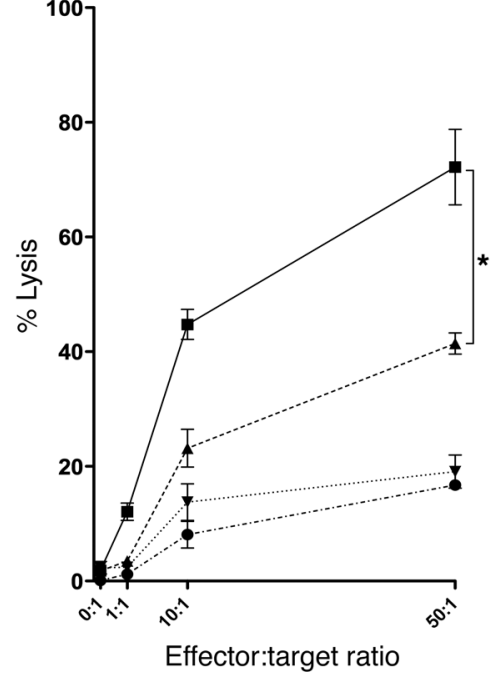

C

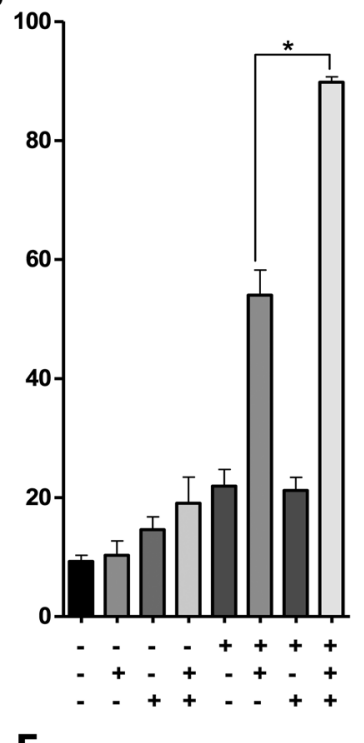

$\mathbf{F}$

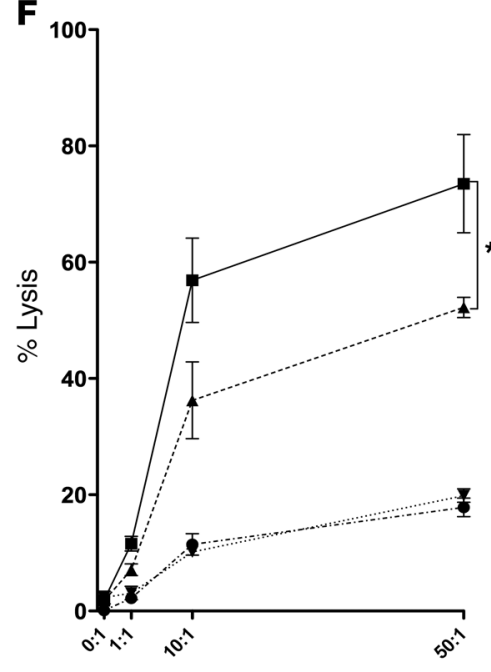

Figure 2

Anti-CD137 agonistic mAb increases cetuximab-mediated NK cell cytotoxicity on tumor cells. To evaluate NK cell function, purified NK cells were isolated from 3 independent, healthy donor PBMCs and cultured for 24 hours together with cetuximab (10 $\mu \mathrm{g} / \mathrm{ml})$ and irradiated (50 Gy) EGFR-expressing cancer cells (PC1) at a ratio of 1:1. After 24 hours, NK cells were isolated by negative selection and assessed for purity (>90\% purity as defined by CD3-CD56+ flow cytometry) and activation (>50\% expression of CD137). EGFR-expressing cancer cell lines including SCC6 ( $\mathbf{A}$ and $\mathbf{D}$ ), PC1 (B and E), and SCC4 (C and F) were cultured for 18 hours with preactivated, purified NK cells in medium alone or with anti-CD137 $\mathrm{mAb}(\mathrm{BMS}-663513,10 \mu \mathrm{g} / \mathrm{ml}$ ) alone, cetuximab $(10 \mu \mathrm{g} / \mathrm{ml})$ alone, or cetuximab plus anti-CD137 mAbs (both at $10 \mu \mathrm{g} / \mathrm{ml})$. Cells were washed and incubated with annexin $\mathrm{V}$ and 7-AAD to determine percentage of apoptotic tumor cells by annexin $\mathrm{V}$ and 7-AAD staining. Chromium-labeled EGFR-expressing cancer cell lines were cultured for 4 hours with preactivated, purified NK cells in medium alone or with anti-CD137 mAb (BMS$663513,10 \mu \mathrm{g} / \mathrm{ml}$ ) alone, cetuximab $(10 \mu \mathrm{g} / \mathrm{ml})$ alone, or cetuximab plus anti-CD137 mAbs. Shown is percentage lysis of target cells by chromium release at varying effector (activated NK cells)/target cell ratios cultured with medium alone (circles), anti-CD137 (upside-down triangle), cetuximab (triangle), or cetuximab and anti-CD137 (square) antibodies. ${ }^{*} P=0.002$, SCC6 (A); ${ }^{*} P=0.007, \mathrm{PC} 1$ (B); ${ }^{*} P=0.007, \mathrm{SCC} 4(\mathbf{C}) ;{ }^{*} P=0.021$, $\operatorname{SCC6}(\mathbf{D}) ;{ }^{*} P=0.011, \mathrm{PC} 1(\mathrm{E}) ;{ }^{*} P=0.049, \operatorname{SCC} 4(\mathbf{F})$.

cetuximab and persisted for as long as 1 week following treatment. Induced expression at the tumor site was greater at all time points compared with that in the spleen or peripheral blood. CD137 expression peaked in the spleen and peripheral blood at 72 hours and returned to baseline by 1 week.

Based on this temporal pattern of CD137 upregulation, antiCD137 agonistic mAb was tested in vivo in the EGFR-expressing xenotransplant model (SCC6) in combination with cetuximab administered concurrently or sequentially with a 24-hour delay. Since athymic mice lack functional $\mathrm{T}$ cells, this model focused on the innate response to the combination of mAbs. A schedule of anti-CD137 mAb given once weekly for 4 weeks had no effect on tumor growth or survival, cetuximab alone had a modest effect, and combination cetuximab and anti-CD137 mAb inhibited tumor growth and prolonged survival (Figure 4, A-C). Sequential, cetuximab followed by anti-CD137 mAb with a 24-hour delay 
A

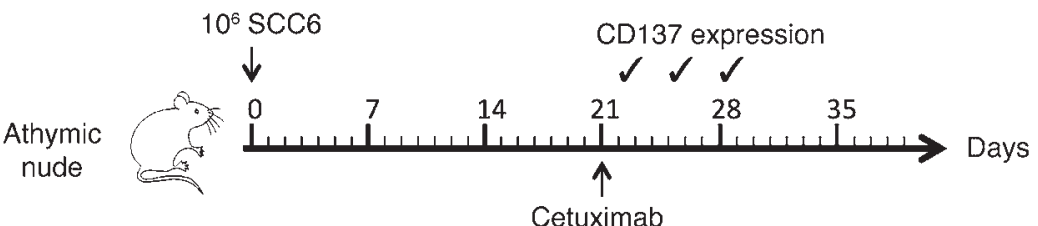

B $\mathrm{CD}^{-}$

Non-tumor bearing

DX5 $5^{+}$Untreated $\quad$ Cetuximab (24 h post)

Splenocytes Splenocytes PBLs
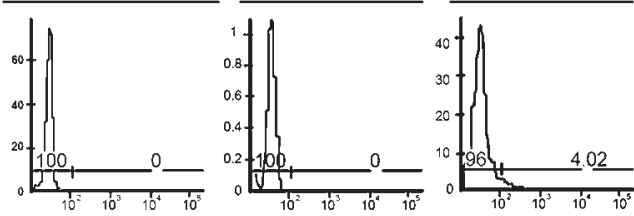

$\uparrow$ Counts

CD137

C $\mathrm{CD}^{-}$

$\mathrm{D} \times 5^{+}$

Tumor bearing
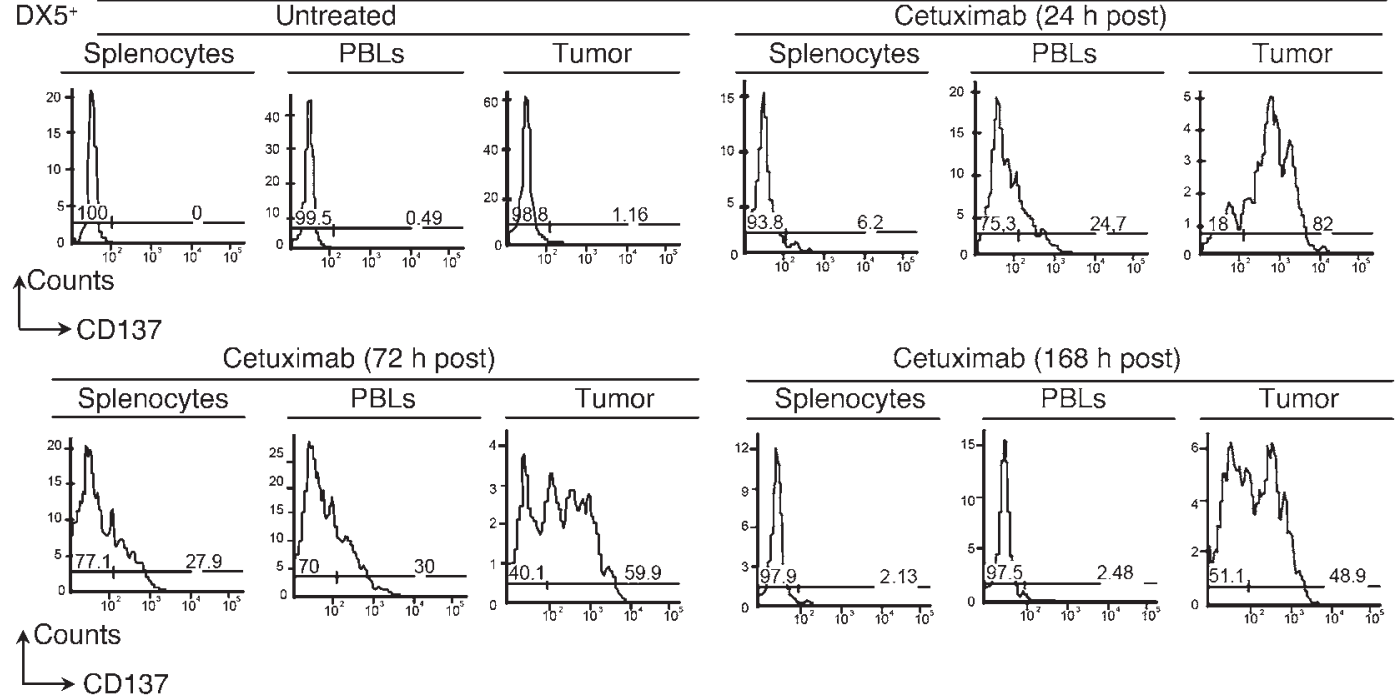

Figure 3

Cetuximab mAb induces CD137 expression in vivo. nu/nu mice were inoculated with $1 \times 10^{6}$ SCC6 tumor cells s.c. on the left flank, and cetuximab was administered on day 21 after tumor inoculation (A, schema). (B) Representative CD137 expression on CD3-DX5+ cells isolated from the spleen or peripheral blood 24 hours following cetuximab treatment or isotype control in non-tumor-bearing mice ( $n=3$ mice per group). (C) Representative CD3-DX5+ cells isolated from the spleen, peripheral blood, or tumor analyzed for CD137 expression 24, 72 , or 168 hours following cetuximab treatment or isotype control in tumor-bearing mice ( $n=3$ mice per group). For $\mathbf{B}$ and $\mathbf{C}$, numbers indicate percentage of cells above and below upper limit of isotype control.

was superior to concurrent combination dosing in effect on both tumor growth and survival. This therapeutic schema was tested on both KRAS-WT (T84) and KRAS-mutant (HCT116 G13D) tumors and demonstrated a similar therapeutic benefit to the sequential combination of cetuximab followed by anti-CD137 mAb, with near complete tumor regression and all animals surviving 100 days after tumor implantation (Figure 4, D-F).

Enhancement of the antitumor activity of cetuximab by anti-CD137 agonistic $m A b$ is dependent on $N K$ cells and $C D 8^{+} T$ cells. The efficacy we observed with combination cetuximab and anti-CD137 $\mathrm{mAb}$ therapy in athymic mice strongly implicates the innate immune system in the mechanism of action. In order to determine whether the adaptive immune response also plays a role, we applied the combination therapy to a syngeneic tumor model in immuno- competent BALB/c mice, using a tumor cell line, TUBO, transfected with human EGFR, previously determined to be sensitive to cetuximab treatment (Figure 5A and ref. 22). This cell line is HER2/neu-dependent and derived from BALB/c mice transgenic for the neu oncogene. In this model, anti-CD $137 \mathrm{mAb}$ therapy alone had no effect on tumor growth and cetuximab monotherapy significantly slowed tumor growth, while the sequential combination of cetuximab followed by anti-CD137 mAb led to tumor regression and improved survival, with all mice in this group alive at day 100 (Figure 5, B and C).

Depletion of $\mathrm{CD}^{+} \mathrm{T}$ cells or macrophages had no influence on efficacy (Figure 5, D and E). As hypothesized, depletion of NK cells almost completely abrogated the therapeutic benefit. But surprisingly, depletion of $\mathrm{CD}^{+} \mathrm{T}$ cells partially reduced the efficacy of the 
A

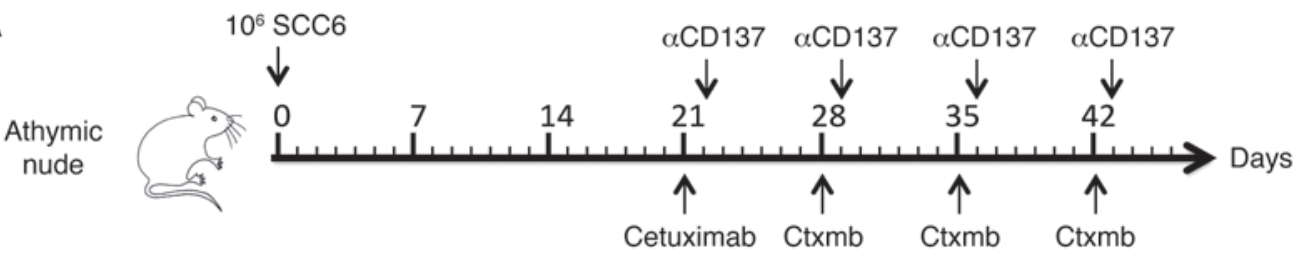

B

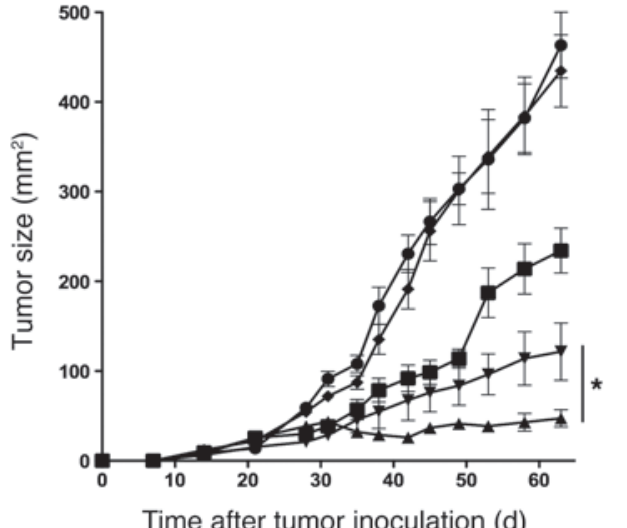

C
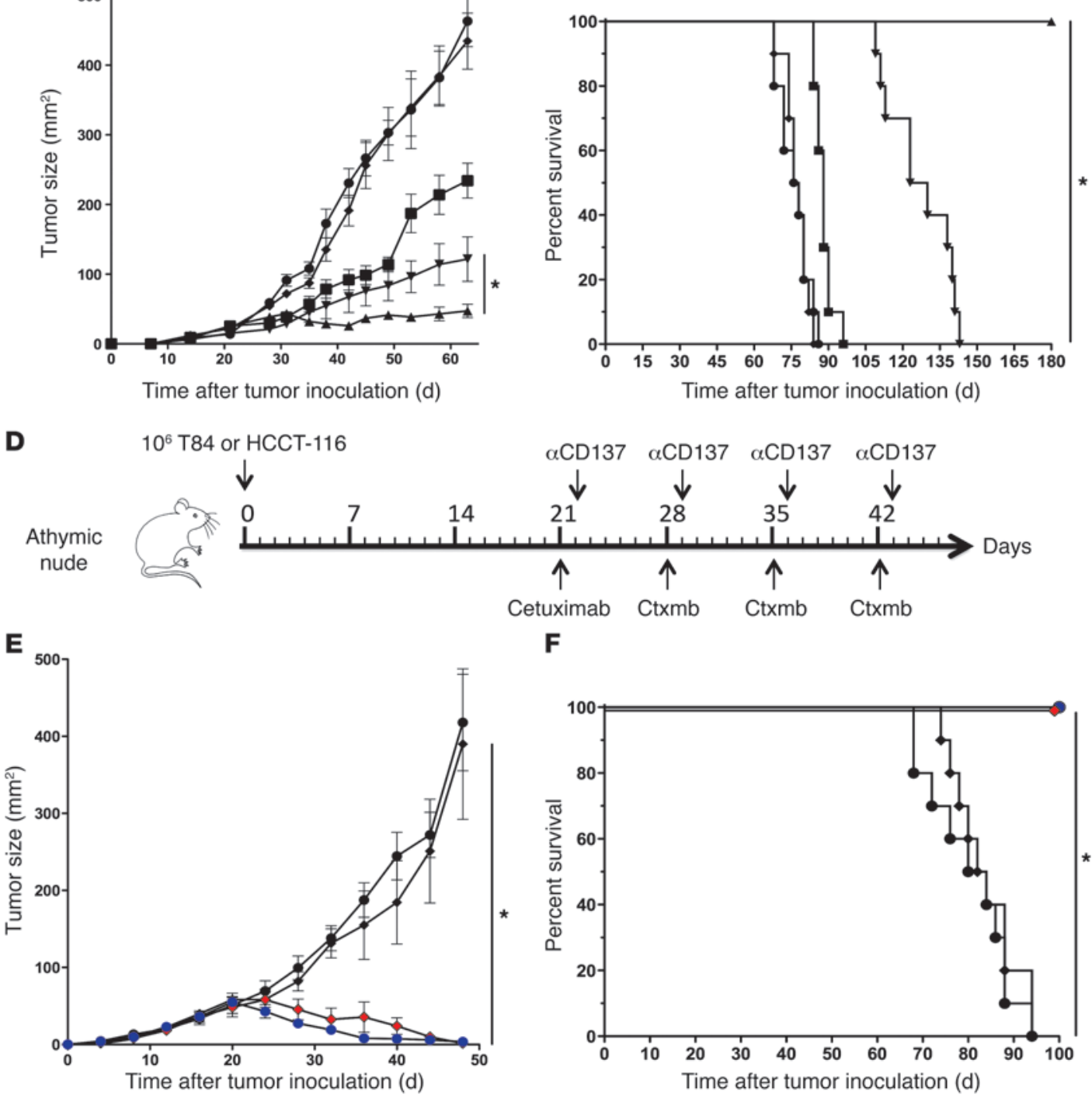

$\begin{array}{lllll}\alpha \mathrm{CD} 137 & \alpha \mathrm{CD} 137 & \alpha \mathrm{CD} 137 & \alpha \mathrm{CD} 137\end{array}$

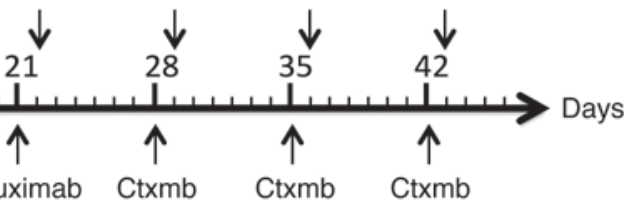

$\mathbf{F}$

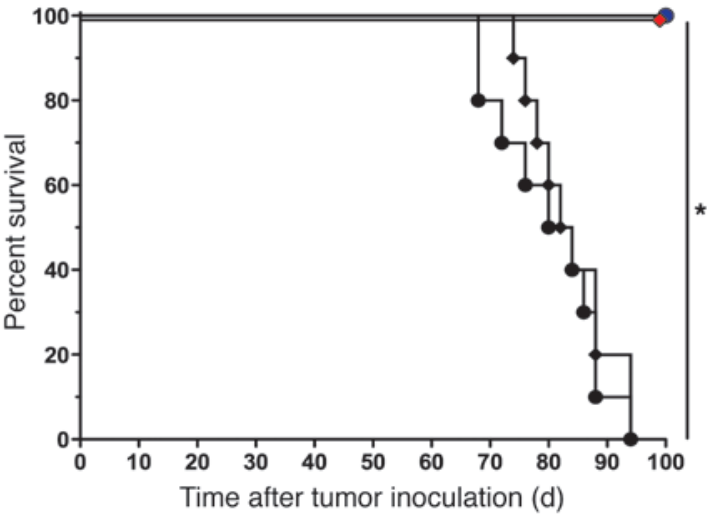

\section{Figure 4}

Anti-CD137 agonistic mAb enhances antitumor activity of cetuximab in vivo against KRAS-mutant and KRAS-WT EGFR-expressing tumors. nu/nu mice were inoculated with $1 \times 10^{6}$ SCC6 tumor cells (KRAS-WT) S.c. on the left flank, and cetuximab and/or anti-CD137 mAb was administered starting on day 21 after tumor inoculation $(\mathbf{A}$, schema). (B and C) Following tumor inoculation on day 0 , mice received either rat IgG control on day 21 (circles), cetuximab on day 21 (squares), anti-CD137 antibody on day 22 (diamonds), cetuximab on day 21 and anti-CD137 antibody on day 21 (upside-down triangles), or cetuximab on day 21 and anti-CD137 antibody on day 22 (triangles), with each injection repeated weekly for a total of 4 injections. Mice (10 per group) were then monitored for tumor growth (B) and overall survival (C). nu/nu mice were inoculated with $1 \times 10^{6}$ T84 (KRAS-WT) or HCT116 (KRAS-mutant) tumor cells s.c. on the left flank, and cetuximab and/or anti-CD137 mAb was administered starting on day 21 after tumor inoculation (D, schema). (E and F) After tumor inoculation, mice inoculated with HCT116 then received either rat IgG control on day 21 (black circles) or cetuximab on day 21 and anti-CD137 antibody on day 22 (blue circles), with each injection repeated weekly for a total of 4 injections. Mice inoculated with T84 then received either rat lgG control on day 21 (black diamonds) or cetuximab on day 21 and anti-CD137 antibody on day 22 (red diamonds), with each injection repeated weekly for a total of 4 injections. Mice (10 per group) were then monitored for tumor growth $(\mathbf{E})$ and overall survival $(\mathbf{F}) .{ }^{*} P<0.001$. 
A
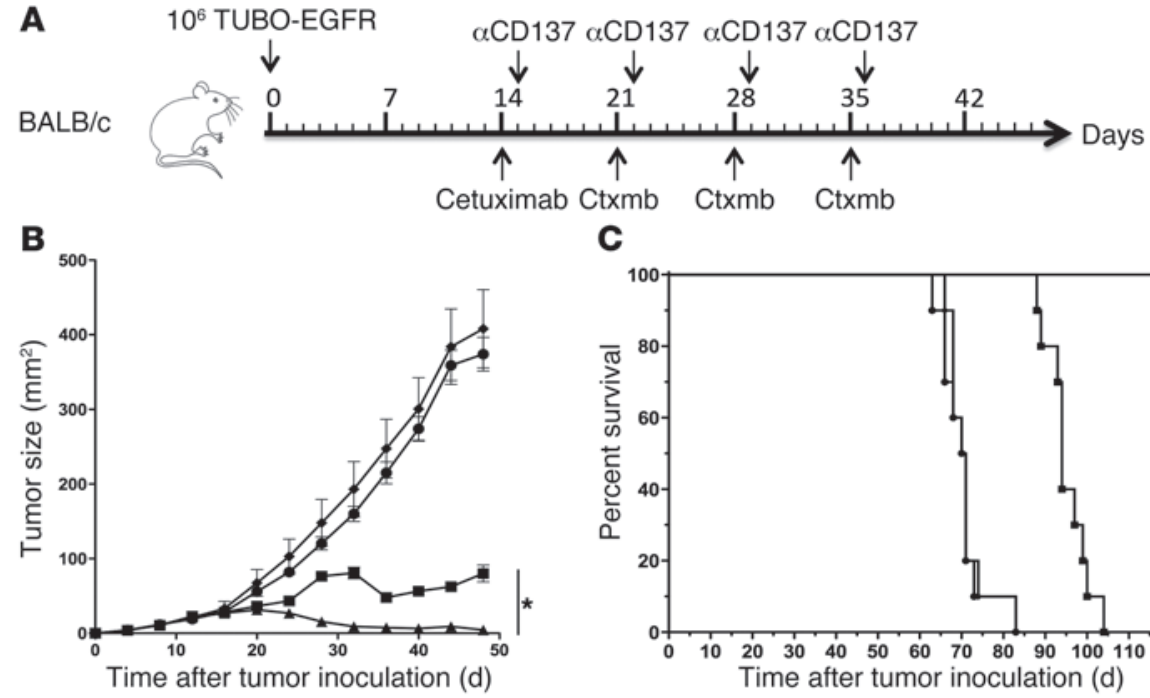

D

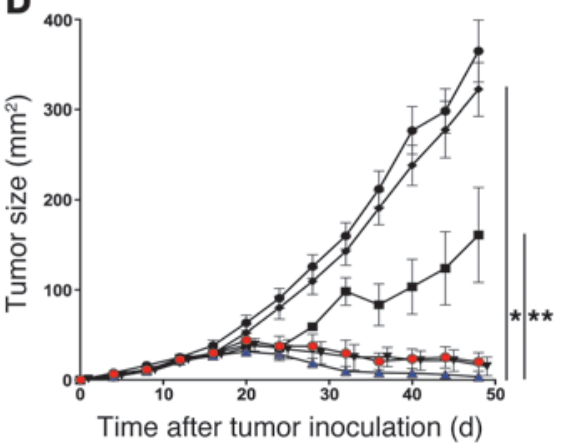

$\mathbf{F}$

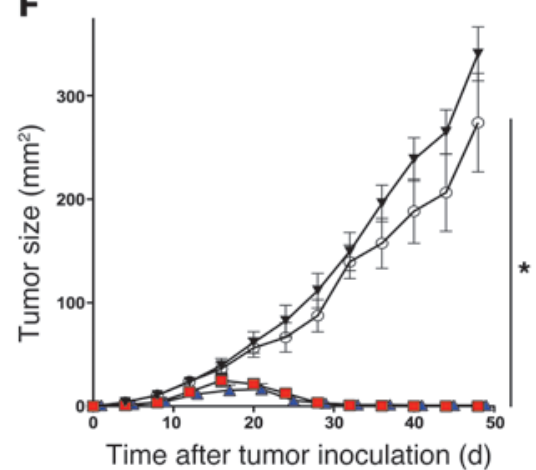

C

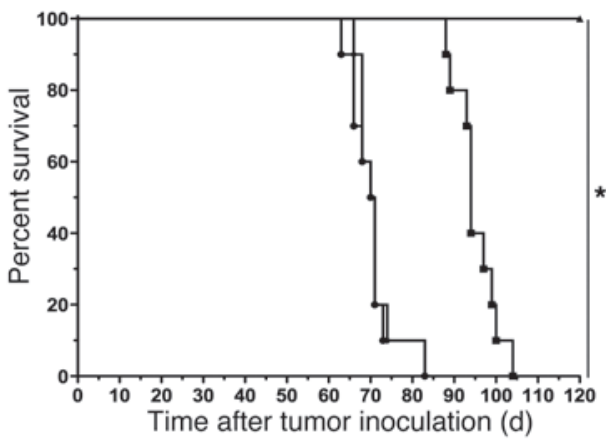

E

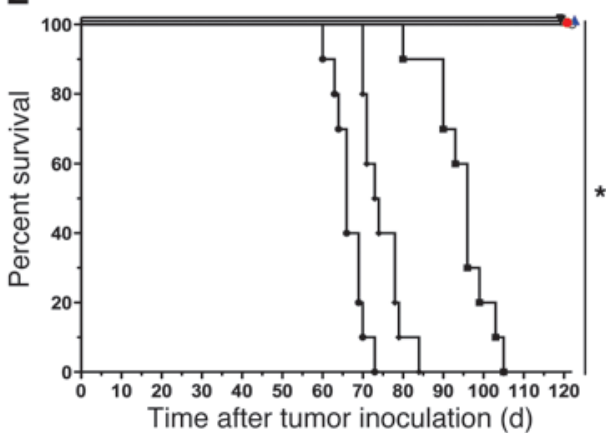

G

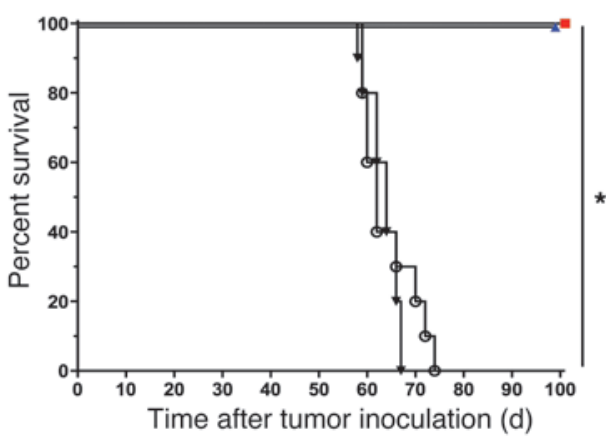

\section{Figure 5}

Enhancement of antitumor activity of cetuximab by anti-CD137 mAb is dependent on NK and CD8+ $\mathrm{T}$ cells. $B A L B / c$ mice were inoculated with $1 \times 10^{6}$ TUBO-EGFR tumor cells s.c. (A, schema). Mice (10 per group) then received either rat IgG on day 14 (circles), cetuximab on day 14 (squares), anti-CD137 antibody on day 15 (diamonds), or cetuximab on day 14 and anti-CD137 antibody on day 15 (triangles), each repeated 4 times weekly, with animals monitored for tumor growth $\left(\mathbf{B},{ }^{*} P=0.047\right)$ and overall survival $\left(\mathbf{C},{ }^{*} P<0.001\right)$. After tumor inoculation, mice (10 per group) received either rat IgG on day 14 (circles) or cetuximab on day 14 and anti-CD137 antibody on day 15 , with each injection repeated weekly for a total of 4 injections with anti-asialo-GM1 starting on day -1 (diamonds), liposomal clodronate starting on day -2 (upside-down triangles), anti-CD8 mAb starting on day -1 (squares), anti-CD4 mAb starting on day -1 (red circles), or no celldepleting $\mathrm{mAb}$ (blue triangles); animals were monitored for tumor growth (D, ${ }^{*} P<0.001$; ${ }^{* *} P<0.008$ ) and overall survival $\left(\mathbf{E},{ }^{*} P<0.001\right)$. Naive BALB/C mice were inoculated with $1 \times 10^{6}$ TUBO-EGFR (circles) or $1 \times 10^{6}$ TUBO tumor cells (upside-down triangles), or BALB/c mice previously (100 days prior) inoculated with TUBO-EGFR tumor cells and cured with cetuximab and anti-CD137 antibody were inoculated with $1 \times 10^{6}$ TUBO-EGFR (blue triangles) or $1 \times 10^{6}$ TUBO tumor cells (red squares) s.c. and monitored for tumor growth $\left(\mathbf{F},{ }^{*} P<0.001\right)$ and overall survival $\left(\mathbf{G},{ }^{*} P<0.001\right)$. combination therapy, suggesting a role for the adaptive immune response in addition to dependence on NK cells. To further investigate adaptive immunity following combination therapy with cetuximab and anti-CD137 mAb, we rechallenged cured mice with both TUBO and TUBO-EGFR tumor cell lines. Mice previously cured with cetuximab and anti-CD137 $\mathrm{mAb}$ rejected both TUBO and TUBO-EGFR (Figure 5, F and G), arguing for epitope spreading and a memory $\mathrm{T}$ cell response. Recognizing an adaptive immune response was present with epitope spreading, we investigated the ability of anti-CD137 mAb to augment the therapeutic immune response in a 2 -tumor model with TUBO and TUBOEGFR tumor cell lines implanted simultaneously on contralateral flanks (Figure 6A). Only in mice receiving the combination of cetuximab and anti-CD137 mAb did both the TUBO and TUBOEGFR tumors regress (Figure 6B). Interestingly, the NK cell and
DC infiltrate within TUBO-EGFR tumors was greater than within TUBO tumors from the same animal; similarly, NK cell and DC infiltrate within TUBO-EGFR tumors was significantly greater in mice receiving anti-CD137 $\mathrm{mAb}$ and cetuximab versus cetuximab alone (Figure 6C). A minor but not significant reduction in $\mathrm{CD}^{+}$ $\mathrm{T}$ cells was observed in both TUBO and TUBO-EGFR tumors in mice receiving the combination versus cetuximab alone. Supporting the enhanced adaptive response and potential mechanism for antitumor activity against a non-EGFR-expressing tumor, the $\mathrm{CD}^{+} \mathrm{T}$ cell infiltrate was less in TUBO or TUBO-EGFR tumors from the mice receiving only cetuximab, while in mice receiving cetuximab and anti-CD137 $\mathrm{mAb}$, the $\mathrm{CD}^{+} \mathrm{T}$ cell infiltrate was greater in both TUBO and TUBO-EGFR tumors. Functionally, circulating $\mathrm{CD}^{+} \mathrm{T}$ cells isolated from mice following cetuximab and anti-CD137 $\mathrm{mAb}$ produced greater IFN- $\gamma$ ex vivo than $\mathrm{CD}^{+}$ 
A
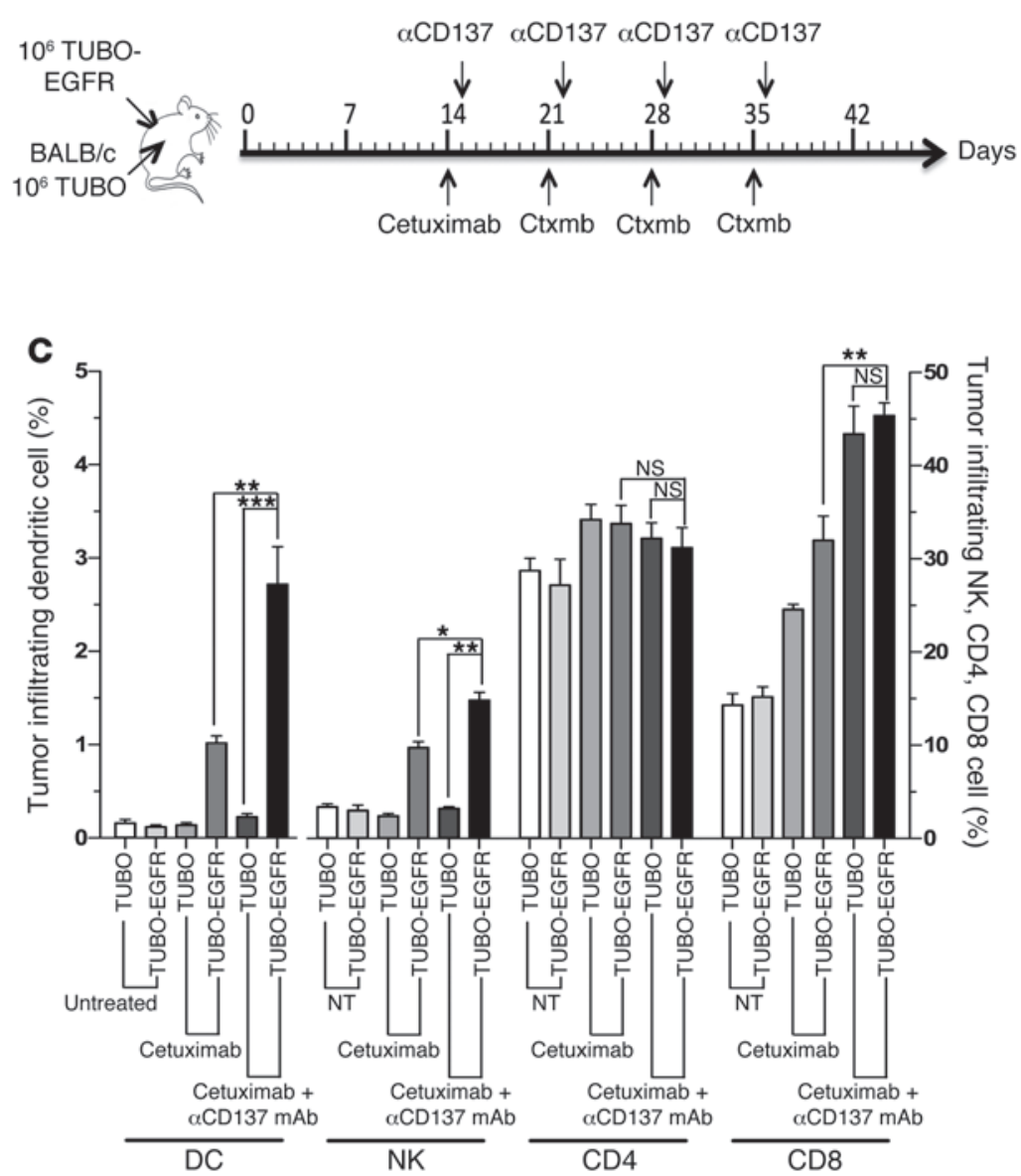

\section{B}

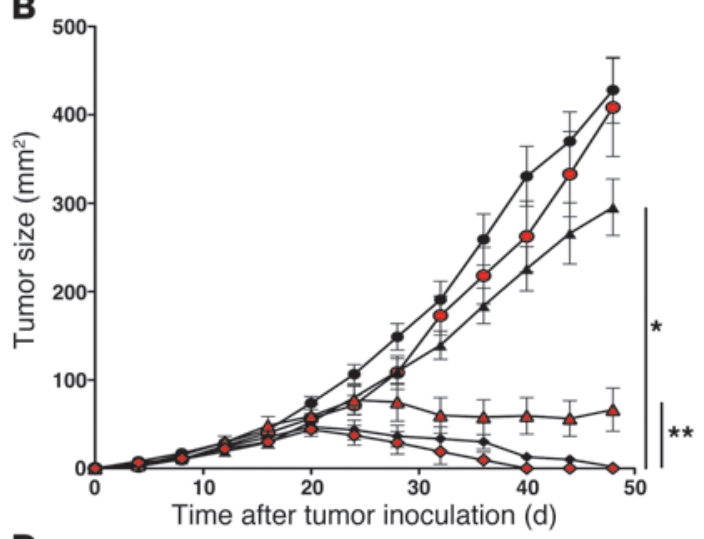

D

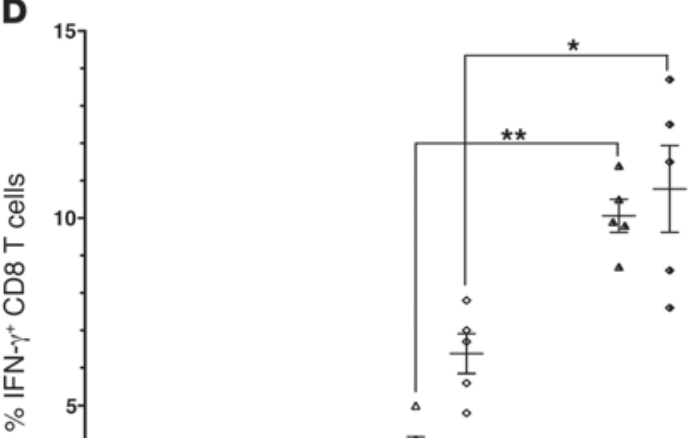

$\frac{7}{T^{2}}$

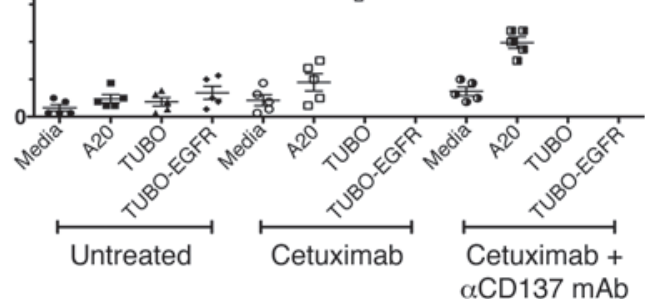

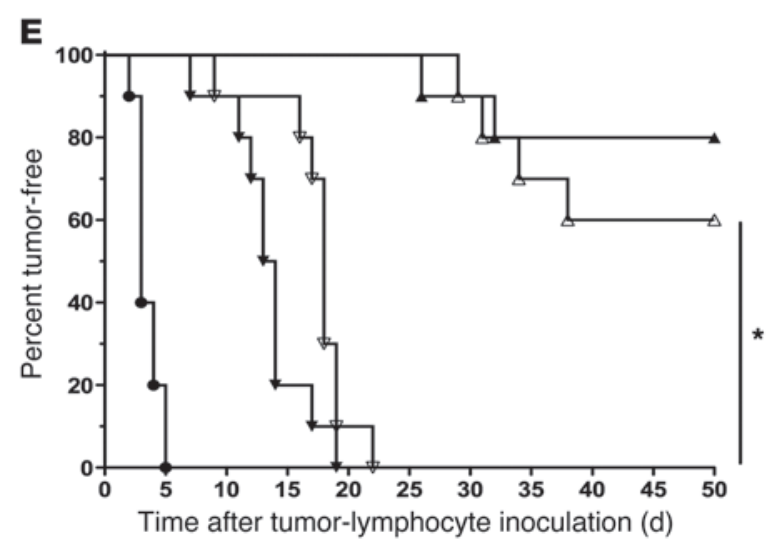

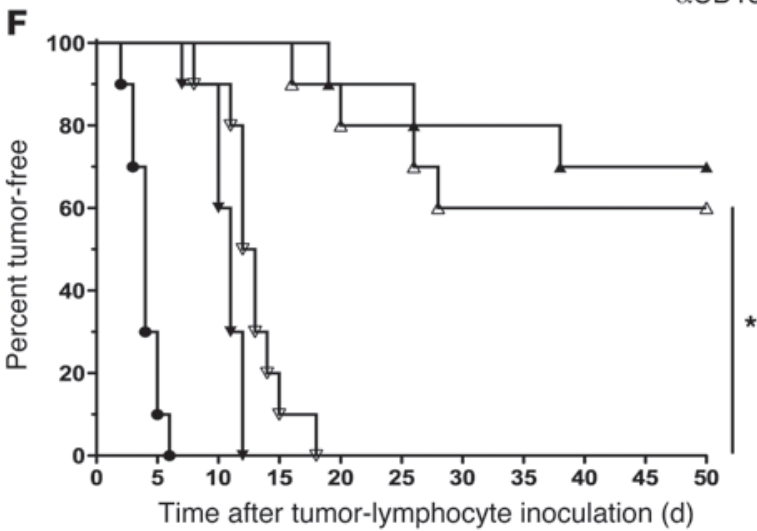




\section{Figure 6}

Anti-CD137 mAb enhances adaptive immunity following cetuximab. BALB/c mice were inoculated with $1 \times 10^{6}$ TUBO-EGFR (red symbols) s.c. on the left flank and $1 \times 10^{6}$ TUBO tumor cells (black symbols) s.c. on the right flank (A, schema). After tumor inoculation, mice (15 per group) then received either rat IgG control on day 14 (circles), cetuximab on day 14 (triangles), or cetuximab on day 14 and antiCD137 antibody on day 15 (diamonds), with each repeated 4 times weekly; animals were monitored for tumor growth $\left(\mathbf{B},{ }^{*} P<0.001\right.$; $\left.{ }^{\star \star} P=0.021\right)$. On day 24 , TUBO and TUBO-EGFR tumors were resected from 5 untreated (NT, IgG control) mice and mice treated with cetuximab monotherapy and combination cetuximab and anti-CD137 mAb; animals were analyzed for percentage of total viable cells representing tumor-infiltrating DCs, NK cells, and $\mathrm{CD}^{+}{ }^{+}$and $\mathrm{CD} 8^{+} \mathrm{T}$ cells $(\mathbf{C}$, $\left.{ }^{\star} P<0.05,{ }^{* *} P<0.01,{ }^{* *} P<0.001\right)$. On day 24 , PBMCs were collected from 5 untreated (black symbols) mice and mice treated with cetuximab monotherapy (white symbols) and combination cetuximab and anti-CD137 mAb (half-black symbols) and analyzed ex vivo for IFN- $\gamma$ production following exposure to medium (negative control, circles), A20 (negative control, squares), TUBO (triangles), or TUBO-EGFR (diamonds) cell lines $\left(\mathbf{D},{ }^{\star} P=0.009,{ }^{\star *} P<0.001\right)$. Splenocytes were obtained from naive mice (total splenocytes, circles) or mice sacrificed 50 days following tumor inoculation and treatment with either cetuximab monotherapy (total splenocytes, CD8-selected, upside-down triangles) or sequential cetuximab and anti-CD137 mAb (total splenocytes, CD8-selected, triangles) and were coinjected (100:1) into naive mice (10 per group) with TUBO $\left(\mathbf{E},{ }^{*} P<0.001\right)$ or TUBO-EGFR $(\mathbf{F}$, $\left.{ }^{*} P<0.001\right)$ and monitored for percentage of mice that were tumor free.

$\mathrm{T}$ cells isolated from mice following cetuximab alone (Figure 6D). Interestingly, $\mathrm{CD}^{+} \mathrm{T}$ cells produced greater IFN- $\gamma$ when exposed to TUBO tumor cells following cetuximab and anti-CD137 mAb therapy compared with cetuximab alone, suggesting anti-CD137 $\mathrm{mAb}$ augments the adaptive $\mathrm{CD}^{+} \mathrm{T}$ cell response with epitope spreading beyond EGFR.

To further compare the relative strength of the adaptive immune response following cetuximab versus combination cetuximab and anti-CD137 mAb therapy, we isolated total and CD8-selected splenocytes from mice previously inoculated with TUBO-EGFR tumor cells and treated them with either cetuximab monotherapy or sequential cetuximab and anti-CD137 mAb combination. As measured by the Winn adoptive transfer assay, anti-CD137 mAb therapy significantly improved adaptive immunity to both TUBOEGFR (Figure 6E) and TUBO (Figure 6F) tumor cells. The majority, $60 \%-80 \%$, of mice were protected against either tumor by total or CD8-selected splenocytes from mice having received combination therapy compared with $0 \%$, no evidence of protection, following transfer of total or CD8-selected splenocytes from mice having received cetuximab monotherapy.

Increased expression of CD137 on circulating and tumor-infiltrating NK cells and increased frequency of EGFR-tetramer ${ }^{+} C D 8^{+}$Tcells in patients receiving cetuximab therapy. We identified 54 patients with HN squamous cell carcinomas receiving cetuximab therapy. PBMCs were isolated prior to and following the first cetuximab administration, including samples obtained every 24 hours for up to 1 week (Figure 7A). A decline occurred in the number of circulating NK cells and in their expression of the FcyRIII, CD16, concomitant with their increased expression of CD137 (Figure 7B). Comparing all pre- to postcetuximab peripheral blood samples, a significant increase in percentage of NK cells expressing CD137 was observed, $4 \%$ to $15 \%$ (Figure 7C), with persistence of upregulated expression of CD137 from 24 hours to 1 week following cetuximab (Figure 7D).
We next investigated the possible influence of Fc $\gamma$ RIIIA polymorphisms on the cetuximab-induced upregulation of CD137 on NK cells. A greater increase in CD137 expression was seen among patients harboring high-affinity alleles Fc $\gamma$ RIIIA-158 (V/V or F/V) compared with those with low-affinity Fc $\gamma$ RIIIA-158 (F/F) alleles (Figure 7E). Finally, we evaluated intratumoral NK cell expression of CD137 in patients with HN cancer and observed a significant increase following cetuximab (Figure 7F).

Taking our observation of an adaptive component to the response to cetuximab and anti-CD137 $\mathrm{mAb}$ together with a prior report demonstrating induction of EGFR-specific $\mathrm{CD}^{+} \mathrm{T}$ cells following cetuximab $(23,24)$, we measured antigen-specific $\mathrm{CD}^{+} \mathrm{T}$ cells by tetramer analysis. A significant increase in EGFR-specific $\mathrm{CD}^{+} \mathrm{T}$ cells was observed approximately 1 week following cetuximab among 10 patients with HN cancer (Figure 8, A and B). To explore the connection between magnitude of innate and adaptive immune response following cetuximab, we tested the correlation between percentage of CD8 ${ }^{+} \mathrm{T}$ cells specific for EGFR and percentage of NK cells upregulating CD137 in peripheral blood in patients following cetuximab therapy. Interestingly, there was a highly significant correlation (Figure 8C).

\section{Discussion}

Activation of EGFR provides a strong growth signal, resulting in tumor proliferation, reduced apoptosis, increased angiogenesis, and a poor prognosis for patients with epithelial cancer, including $\mathrm{CRC}$, and $\mathrm{HN}$ squamous cell as well as pancreatic and non-small cell lung cancer $(25,26)$. Despite extensive study, including 17 randomized trials enrolling nearly 8,000 patients, cetuximab therapy confers an improved response rate and progression-free and overall survival only in HN carcinoma and in KRAS-WT CRC (6).

Here, we report a method to enhance the efficacy of cetuximab therapy. In contrast to efforts to improve the single agent efficacy of a mAb, such as by increasing $\mathrm{Ab}$ affinity, making versions with 2 different specificities, conjugation to toxins, or conjugation to radio-isotopes, here we propose to augment the immune response following administration of the tumor-targeted $\mathrm{mAb}$ by using a sequential combination of 2 different antibodies. First, cetuximab localizes to the site of the tumor and activates surrounding NK cells via the $\mathrm{Fc}-\mathrm{Fc} \gamma \mathrm{R}$ interaction, thereby increasing NK cell expression of activation and costimulatory markers, such as CD137. An agonistic mAb against CD137 is then administered to enhance the activated NK cell's ability to kill cetuximab-coated tumor cells.

Our findings here with cetuximab are consistent with our results in 2 prior models, including rituximab and trastuzumab, targeting CD20 and HER2/neu, respectively, underscoring the general applicability of this approach $(27,28)$. Prior literature supports that CD137, also known as 4-1BB, is expressed on activated CD4+ and $\mathrm{CD}^{+} \mathrm{T}$ cells, and following trimerization on the cell surface, it provides a proliferative T cell signal (29). Recently, CD137 expression has also been reported on monocytes, DCs, and nonhematopoietic cells with potential functional importance (30,31). CD137 ligand expression, as observed in acute myeloid leukemias, interestingly has been proposed to lead to an inhibitory signal (32) in which leukemic blasts have been shown to impair NK cell spontaneous cytotoxicity. The function of anti-CD137 mAb could, therefore, block an inhibitory signal from the tumor cell to the incoming NK cell in tumors that express the CD137 ligand. Importantly, we did not observe CD137 ligand expression on the HN or CRCs investigated in this study, arguing against this mechanism for the 
A

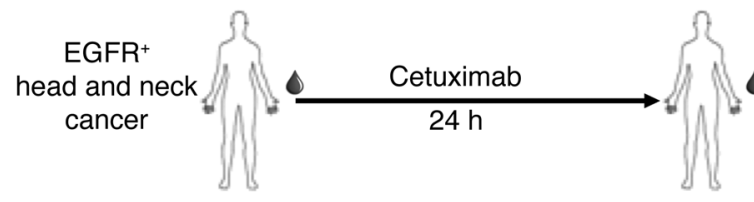

B

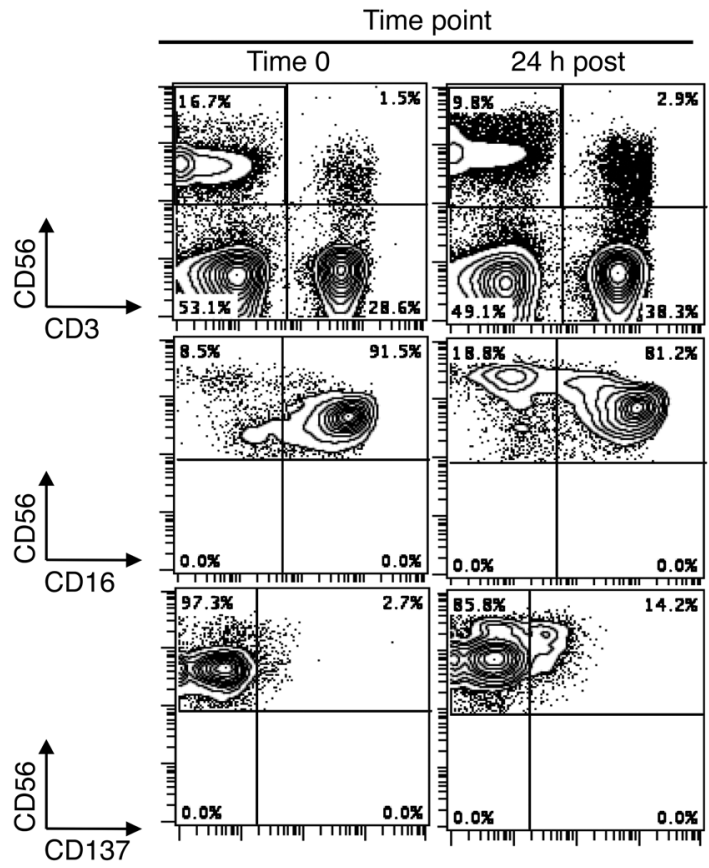

E

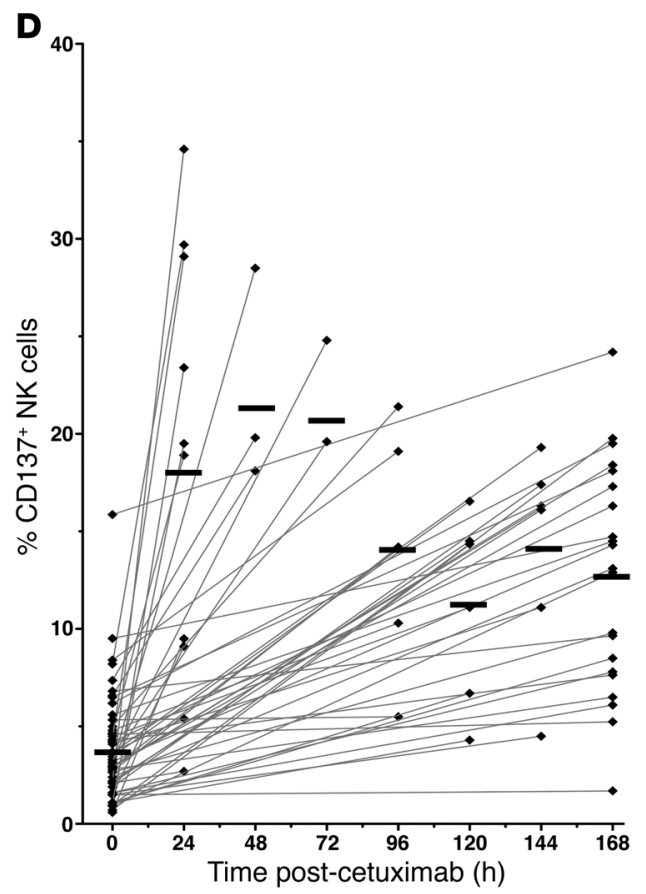

Circulating and

- intratumoral NK cell CD137 expression

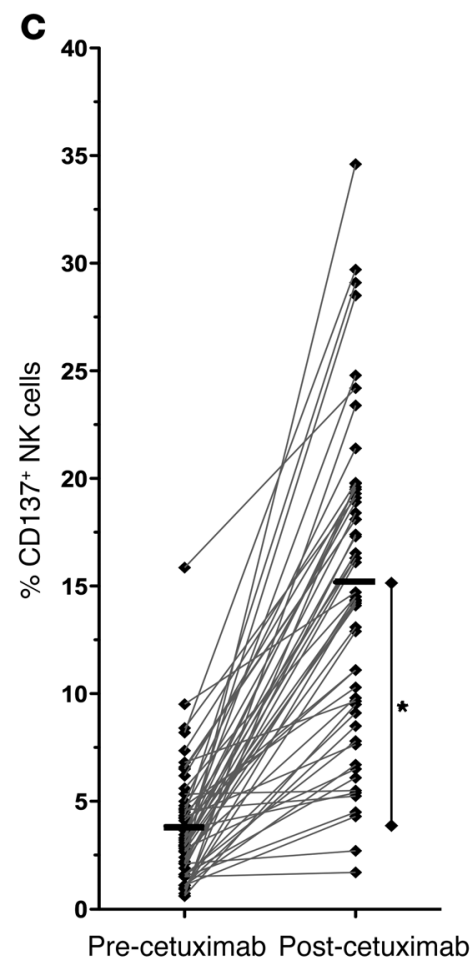

F

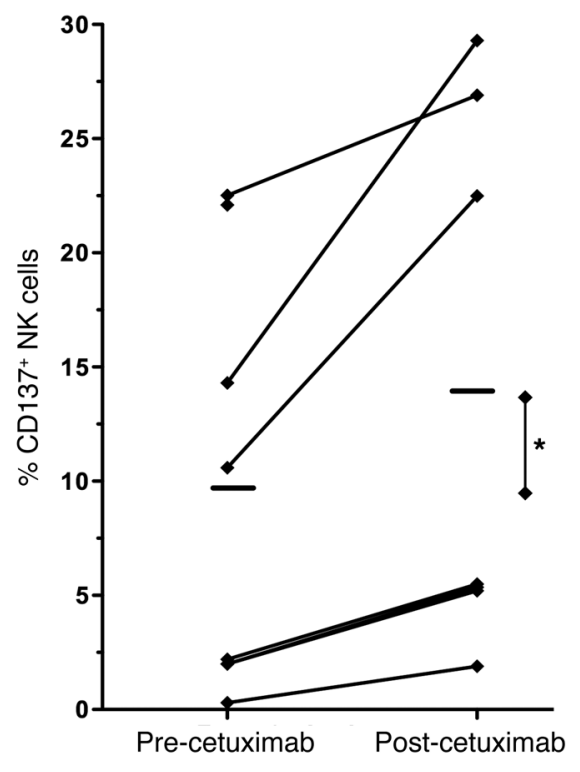

FCyRIIIA-158 polymorphism 


\begin{abstract}
Figure 7
Increased expression of CD137 on circulating and tumor-infiltrating NK cells in patients receiving cetuximab therapy. PBMCs or tumor biopsies by fine needle aspirate (FNA) were isolated from 54 patients with EGFR-expressing HN carcinoma (A). (B) CD56 and CD3 expression on circulating lymphocytes and CD16 and CD137 expression on NK cell subsets CD3-CD56 bright and CD3-CD56 dim from a single representative patient at 2 time points, including prior to cetuximab and 24 hours following cetuximab (number indicates percentage of cells as divided by quadrant). (C) Percentage of CD137 ${ }^{+}$cells among circulating CD3-CD56 ${ }^{+} \mathrm{NK}$ cells from 54 patients prior to and following cetuximab (mean at each time point denoted by bar, ${ }^{*} P<0.001$ ). (D) Percentage of CD137+ cells among circulating CD3-CD56+ NK cells from 54 patients prior to and following cetuximab stratified by specific time after cetuximab infusion (mean at each time point denoted by bar). (E) Absolute change in percentage of $\mathrm{CD} 137^{+}$cells among circulating CD3-CD56 ${ }^{+}$NK cells from 54 patients prior to and following cetuximab stratified by FcyRIIIA-158 polymorphism $(P=0.011)$. (F) Percentage of $\mathrm{CD} 137^{+}$cells among tumor-infiltrating CD3-CD56+ NK cells from 8 patients prior to and following cetuximab (1 patient with only precetuximab sample and no postcetuximab sample evaluable; $P=0.020$ ).
\end{abstract}

enhanced NK cell function. Our observation is consistent with additional mouse and human studies that report anti-CD137 enhancement of NK cell killing in both hematopoietic and solid tumors lacking expression of the CD137 ligand (33-36). Rather, by first activating NK cells via CD16 and the Fc-Fc $\gamma$ R interaction, surface expression of CD137 is induced and subsequently directly stimulated by administering the agonistic anti-CD137 mAb.

Although CD137 stimulation has been shown to enhance NK cell function and proliferation in mice (33-36), a recent study suggests that it may have opposite effects on human NK cells, leading to decreased function, including cytotoxicity (32). This discrepancy with our current results is likely due to differences in experimental conditions. First, we used freshly isolated NK cells instead of NK cells cultured with IL-2 and feeder cells for 10 days. Second, we stimulated CD137 using an agonistic antibody as opposed to the CD137 ligand (4-1BBL). Finally, we measured enhancement of ADCC as opposed to natural spontaneous NK cell cytotoxicity.

To directly implicate NK cells as the main effector cells responsible for augmenting cetuximab, we used purified NK cells for in vitro studies. For in vivo studies, we used tumors engrafted into nu/nu mice, which have NK cells, macrophages, and complement, but lack $T$ cells, and in addition, we used syngenic mouse models that were depleted in various cell subsets. NK cell expression of CD137 has been observed in prior studies using immobilized IgG1 or rituximab-coated lymphoma cells $(21,37)$. Expression occurs predominantly among the CD56 $6^{\mathrm{dim}}$ subset of NK cells known to mediate ADCC compared with CD56 ${ }^{\text {hi }} \mathrm{NK}$ cells, which are responsible for secretion of cytokines such as IFN- $\gamma$ (38), explaining our result of a lack of increased IFN- $\gamma$ with anti-CD137 mAb despite enhanced ADCC. In the preclinical models and patient samples following cetuximab therapy, we observed a concurrent downregulation of CD16 among the CD56 ${ }^{\mathrm{dim}}$ subset, supporting the internalization of the Fc $\gamma \mathrm{R}$ following Fc-Fc $\gamma \mathrm{R}$ binding $(39,40)$.

A potential advantage of the combination strategy we propose is that only the NK cells in contact with cetuximab-bound tumor cells will be activated, as opposed to other more general NK cell-activating strategies, such as high-dose IL-2, IL-12, or other cytokines, which though clinically beneficial, are limited by the systemic toxicity (41-44). Our more directed NK activation would occur wherever tumor cells are located, regardless of anatomic site, such as within the primary tumor, tumor-involved lymph nodes, or at distant sites of metastatic cancer. We did observe activated NK cells in circulation of patients as well as in the spleen of mice following cetuximab. We presume that these activated NK cells had migrated from their initial site of antibody-induced activation in the tumor. Consistent with this view is our finding that in patients, the highest and most prolonged upregulation of CD137 was noted on intratumoral NK cells.

Despite the predominant role of NK cells and the innate immune response underlying the mechanism of synergy, we observed a role for $\mathrm{CD}^{+} \mathrm{T}$ cells in the antitumor response. In the combination of anti-CD20 mAb followed by anti-CD137 mAb, increased late relapses were noted when $\mathrm{CD}^{+} \mathrm{T}$ cells were depleted (45). In the current study, $\mathrm{CD}^{+} \mathrm{T}$ cell depletion abrogated the survival benefit and reduced the initial antitumor response. Interestingly, however, in our rechallenge experiment, we observed protection not only against an EGFR-expressing cell line, but also the identical cell line without EGFR expression, suggesting epitope spreading occurred and antitumor memory developed that was not limited to EGFR. In a prior immunocompetent mouse model of lymphoma, targeted by anti-CD20 mAb, protection from rechallenge was demonstrated; however, the model did not allow a determination of whether an immune response extended beyond CD20 (46). In further support by the Winn assay (47), we observed that anti-CD137 $\mathrm{mAb}$ following cetuximab treatment enhanced adaptive immunity with a significantly higher rate of $\mathrm{CD}^{+} \mathrm{T}$ cell-mediated protection to EGFR-expressing and nonexpressing tumors. The role of adaptive immunity and $\mathrm{CD}^{+} \mathrm{T}$ cells may be an on-target effect of anti-CD137 mAbs in vivo influencing the function of multiple cell types including hematopoietic cells such as granulocytes, $\mathrm{T}$ cells, B cells, DCs, and monocytes in addition to NK cells, as well as nonhematopoietic cells $(31,48-51)$. Recently, Lee et al. reported that tumor-targeting $\mathrm{mAbs}$ such as cetuximab induce an adaptive tumor antigen-specific $\mathrm{T}$ cell response that is primed by NK cell activation and subsequent NK cell-DC crosstalk (20). Our in vitro observations of enhanced IL-12, IFN- $\gamma$, and TNF- $\alpha$ support these findings that activated human NK cells increase DC maturation and cytokine production in both a cell-contact-dependent, due to NKp30, and independent manner that can be further enhanced by anti-CD137 $\mathrm{mAb}$ stimulation $(52,53)$. In vivo, the addition of anti-CD137 mAb to cetuximab increases recruitment of NK cells as well as DCs to EGFR-expressing tumors, augmenting the innate immune response locally and secondarily enhancing DC function and adaptive immunity with increased local and distant tumor infiltration with $\mathrm{CD}^{+} \mathrm{T}$ cells.

The systemic nature of the $\mathrm{T}$ cell response observed in the mouse model is consistent with findings in the peripheral blood of patients with HN carcinoma. Circulating EGFR-specific T cells have been identified that have preserved proliferative and cytotoxic function as well as a direct correlation with levels of EGFR expression on the tumor and size of the EGFR-specific $T$ cell population (23). Recently, the NK cell-DC interaction has been reported to promote cross-presentation of tumor antigen independently of NK cell cytotoxicity, (54) and Srivastava et al. connected these findings by identifying enhanced cross-presentation leading to EGFRspecific T cells following NKG2D/MICA binding and induction of DC maturation among patients receiving cetuximab therapy (24). Therefore, as our results demonstrate, anti-CD137 mAb stimulation enhances NK cell-DC crosstalk while also directly enhancing 
A

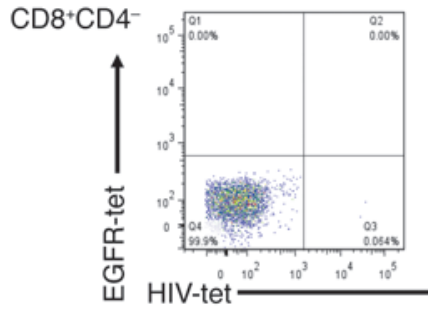

Healthy control 1

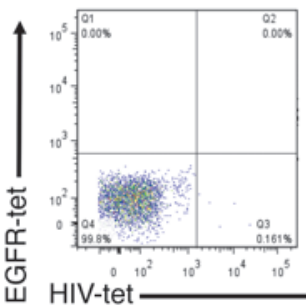

Healthy control 2

B

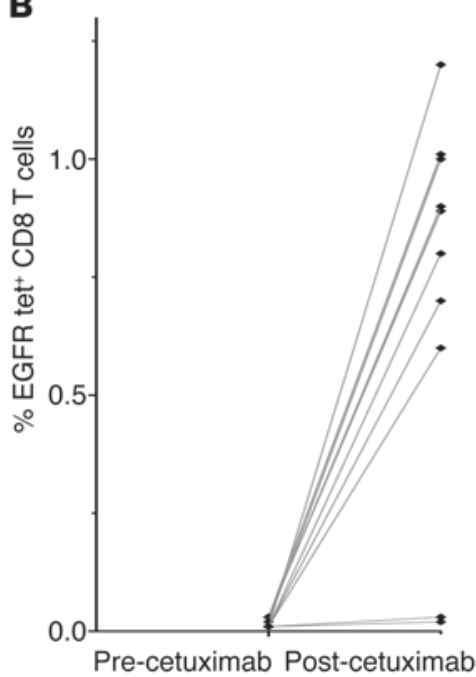

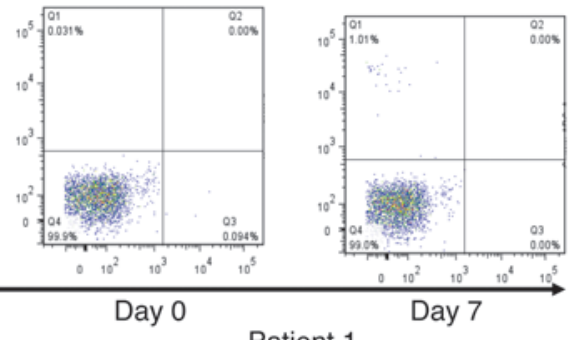

Patient 1

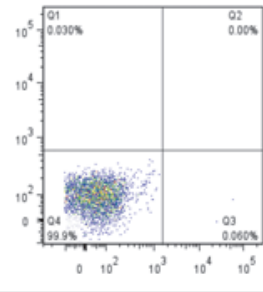

Day 0

C

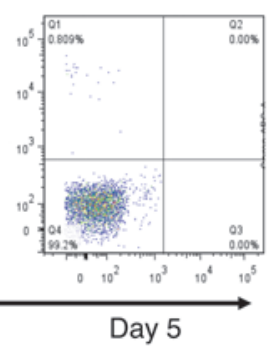

Patient 2

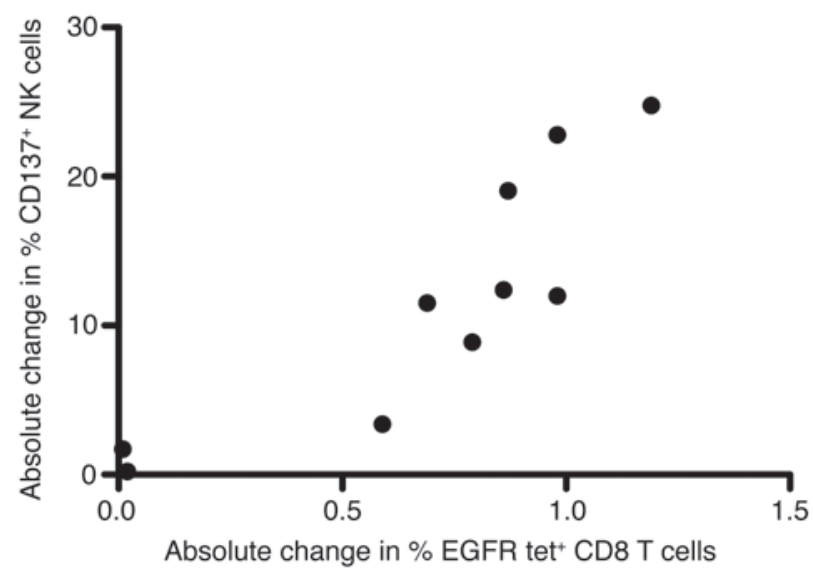

Figure 8

Increased frequency of EGFR-tetramer+ $\mathrm{CD}^{+} \mathrm{T}$ cells in patients receiving cetuximab therapy. (A) HIV-tetramer-specific or EGFR-tetramer-specific (YLN) CD8+ T cells from 2 healthy controls and 2 representative patients with HN cancer prior to and 7 or 5 days following cetuximab therapy (number indicates percentage of cells as divided by quadrant). (B) Percentage of EGFR-tetramer-specific CD8 ${ }^{+} \mathrm{T}$ cells from 10 patients with HN cancer prior to and 5 to 7 days following cetuximab therapy. (C) Correlation of absolute change in percentage of CD137+ cells among circulating CD3-CD56 ${ }^{+}$NK cells and absolute change in percentage of EGFR-tetramer-specific CD8 ${ }^{+} \mathrm{T}$ cells from 10 patients with $\mathrm{HN}$ cancer prior to and following cetuximab therapy $\left(R^{2}=0.87, P=0.001\right)$.

NK cell and $\mathrm{CD}^{+} \mathrm{T}$ cell function, particularly NK cell cytotoxicity and $\mathrm{CD}^{+} \mathrm{T}$ cell production of IFN- $\gamma(20,55-57)$. Based on these findings, we selected a series of HLA-A2 ${ }^{+}$patients with HN carcinoma and assayed for presence of EGFR-specific $\mathrm{CD}^{+} \mathrm{T}$ cells. We observed not only presence and expansion of this population following cetuximab treatment, but also a direct correlation, with increase in EGFR-specific T cells and increase in CD137 expression on NK cells. Our results further support the tight interplay between the innate and adaptive immune response following cetuximab therapy. We hypothesize that anti-CD137 agonistic $\mathrm{mAb}$ therapy may both stimulate ADCC due to cetuximab-activated NK cells and promote the proliferation and cytotoxicity of EGFR-specific $\mathrm{T}$ cells induced by cetuximab treatment.
The addition of anti-CD137 mAb therapy to cetuximab shows promise of improving its efficacy against $\mathrm{HN}$ and CRC and also, perhaps, extending its efficacy to other EGFR-expressing cancer types. KRAS mutations confer increased resistance to the ability of cetuximab to block phosphorylation and signal transduction, but do not confer resistance to ADCC (58). Therefore, we investigated whether the addition of anti-CD137 agonistic mAb stimulation could extend cetuximab to KRAS-mutant tumors. We observed equal efficacy in reducing tumor growth and prolonging survival with the combination of cetuximab and anti-CD137 mAb therapy against KRAS-WT and KRAS-mutant tumors. Enhancing ADCC with CD137 stimulation may offer the opportunity to overcome this important mechanism of tumor resistance. 
We have studied patients with HN carcinoma receiving therapy with cetuximab. We found NK cells in circulation and in the tumors that have upregulated CD137. We have also found that Fc $\gamma$ RIII polymorphisms correlate with the magnitude of CD137 upregulation, with increased CD137 associated with the highaffinity alleles. We observed a significant increase in NK cell CD137 expression following cetuximab occurring within 24 hours; in some patients, it was elevated as long as 1 week. The increased expression in the peripheral blood and within the tumor was heterogeneous, with a subset of patients demonstrating minimal upregulation. Though the ongoing and planned phase I and I/II clinical trials do not limit enrollment based on CD137 expression, we speculate that patients with minimal upregulation of CD137 may not benefit from anti-CD137 mAb therapy. To identify predictors of CD137 upregulation, the Fc $\gamma$ RIII polymorphism of all patients was typed and stratified by presence of a single high-affinity allele. Patients with $\mathrm{HN}$ cancer with either $\mathrm{V} / \mathrm{V}$ or V/F demonstrated greater CD137 upregulation compared with patients with 2 low-affinity alleles. With continued enrollment in this phase 0 study, we will identify clinical and pathologic characteristics that predict the heterogeneity in CD137 upregulation. By doing so, we hope to create a nomogram predictive of CD137 expression, which with validation in ongoing clinical trials and correlation with clinical response to anti-CD137 mAb therapy, may provide a predictive biomarker for patient selection and individually tailored treatment schedules.

By changing the paradigm of improving antibody technology not by creating superior tumor-targeting $\mathrm{mAbs}$, rather by targeting the secondary immune response - we have identified a therapeutic strategy that is selective to the tumor microenvironment and efficacious in multiple models, including those resistant to treatment. The approach is dependent upon sequential targeting of first, the tumor, by cetuximab, and second, the activated NK cells, by anti-CD137 agonistic mAbs. This strategy initiates a more robust innate immune response with augmented ADCC as well as adaptive immunity through likely enhanced crosstalk between NK cells, DCs, and tumor antigen-specific CD8 ${ }^{+} \mathrm{T}$ cells. By investigating predictors of CD137 expression, such as Fc $\gamma$ RIII polymorphism, in patients receiving cetuximab monotherapy, we hope to identify biomarkers that will be tested along with the central hypothesis of this new strategy in a planned combination trial with an anti-CD137 agonistic antibody.

\section{Methods}

Cell lines and culture. The human EGFR-expressing cancer cell lines SCC4, SCC6, and PC1 were obtained from John Sunwoo at Stanford University. SCC4 is an upper aerodigestive tract squamous cell carcinoma with mutations in CDKN2A, NF1, and TP53 and WT KRAS, EGFR, MET, NRAS, and $P T E N$. SCC6 is an upper aerodigestive tract squamous cell carcinoma with mutations in CDKN2A and WT NF1, TP53, KRAS, EGFR, ET, NRAS, and $P T E N$. PC1 is a pancreatic adenocarcinoma with mutations in CDKN2A, KRAS (G12D), and TP53 and WT EGFR, NRAS, MET, NF1, and PTEN. Human EGFR-positive cancer cell lines T84 (KRAS-WT), HCT116 (KRAS $G 13 D$ mutant), and A20 (a lymphoma cell line used as a negative control) were obtained from ATCC. T84 is a CRC with mutations in APC and TP53 and WT NF1, KRAS, NRAS, MET, PTEN, and EGFR. HCT116 is a CRC with mutations in KRAS (G13D), APC, and TP53 and WT NF1, NRAS, MET, $P T E N$, and EGFR. The TUBO-EGFR murine cancer cell line was obtained from Yang-Xin Fu at the University of Chicago. The SCC4, SCC6, PC1, and T84 cell lines were cultured in a 1:1 mixture of DMEM:F12 supplemented with $10 \%$ heat-inactivated FCS (HyClone Laboratories), 2\% L-glutamine, $1 \%$ penicillin, and $1 \%$ streptomycin (all from Invitrogen Life Technologies). The HCT116 cancer cell line was cultured in DMEM medium (Invitrogen Life Technologies) supplemented as above. The TUBO-EGFR cancer cell line was cultured in DMEM supplemented with 10\% FCS, 10 mM Hepes, $1 \%$ nonessential amino acids, $1 \%$ penicillin, and $1 \%$ streptomycin (all from Invitrogen Life Technologies). The A20 cell line was cultured in RPMI 1640 medium supplemented with $10 \%$ heat-inactivated FCS (HyClone Laboratories), 2\% L-glutamine, $1 \%$ penicillin, and 1\% streptomycin (all from Invitrogen Life Technologies) and $50 \mu \mathrm{M} 2-\mathrm{ME}$ (Gibco; Invitrogen). Cells were grown as adherent cultures at $37^{\circ} \mathrm{C}$ in $5 \% \mathrm{CO}_{2}$ and passaged after detachment by $0.05 \%$ trypsin (Invitrogen Life Technologies). SCC6, PC1, SCC4, T84, HCT116, and TUBO-EGFR express moderate to high levels of EGFR with specific fluorescence indices (tumor MFI/isotype MFI) of 17.2, 18.1, 53.1, 55.2, 56.1, and 63.3. These cell lines express neither the CD137 ligand nor the CD137 receptor.

Mice. Five- to six-week-old female athymic $(n u / n u)$ nude-Foxn $1^{n u}$ and BALB/C mice were purchased from Harlan and Jackson Laboratories and were housed at the Laboratory Animal Facility at Stanford University Medical Center.

Therapentic antibodies. Rat anti-mouse CD137 (4-1BB) mAb (IgG2a, clone $2 \mathrm{~A}$; ref. 59) was produced from ascites in SCID mice as previously described $(31,45)$. Control rat IgG was obtained from Sigma-Aldrich. Human antihuman CD137 mAb (BMS-663513, IgG4) was provided by Bristol-Myers Squibb. Rituximab (murine-human chimeric anti-CD20, IgG1) and cetuximab (murine-human chimeric anti-EGFR, IgG1) were obtained from Genentech and Bristol-Myers Squibb.

CD137 expression on human NK cells and EGFR-tetramer ${ }^{+} C D 8^{+} T$ cells from patient samples and healthy individuals. PBMCs were isolated from patients with $\mathrm{HN}$ cancer at multiple time points and from healthy donors obtained from the Stanford Blood Center by density gradient separation using Ficoll-Paque Plus (Amersham Biosciences). PBMCs from patients with $\mathrm{HN}$ cancer at time points including immediately prior to and either 24 , $48,72,96,120,144$, or 168 hours after cetuximab infusion were assayed for NK cell expression of CD137 and CD16 as well as EGFR-tetramer, HIVtetramer, and CD8 in a subset of patients. PBMCs isolated from healthy individuals or purified NK cells isolated by negative magnetic cell sorting using NK cell isolation beads (Miltenyi Biotec) were cultured at a ratio of 1:1 in complete medium for 24 hours with breast cancer cell lines in the presence of rituximab $(10 \mu \mathrm{g} / \mathrm{ml})$ or cetuximab $(10 \mu \mathrm{g} / \mathrm{ml})$. Assessment of CD137 and CD16 expression on NK cells was performed in triplicate for each of 3 healthy individuals.

In vitro NK functional assays. PBMCs or purified NK cells were incubated for 24 hours with irradiated (50 Gy) EGFR-expressing cancer cells (SCC6, PC1, or SCC4) at a ratio of 1:1 and with cetuximab $(10 \mu \mathrm{g} / \mathrm{ml})$. Following 24-hour culture of PBMCs with irradiated cancer cells, purified NK cells were then isolated by negative magnetic cell sorting using NK cell isolation beads (Miltenyi Biotec), according to the manufacturer's instructions, at more than $90 \%$ purity, as defined by $\mathrm{CD} 3^{-} \mathrm{CD} 56^{+}$, confirmed by flow cytometry. Activation of NK cells was confirmed by flow cytometry (>50\% expression of CD137). The activated NK cells were then cultured with additional EGFR-expressing cancer cell lines (SCC6, PC1, or SCC4) at a ratio of 1:1. After 18 hours, the supernatants were removed and assayed for IFN- $\gamma$ by ELISA (Human Interferon- $\gamma$ BD OptEIA ELISA Set and BD OptEIA Reagent Set B; BD Biosciences - Pharmingen). Cells from these cultures were incubated with FITC-annexin V and 7-AAD (both BD Biosciences) and analyzed by flow cytometry. Forward and side scatter were used to gate out the NK cells, and fluorescence was used to determine the percentage of apoptotic tumor cells by annexin $\mathrm{V}$ and 7-AAD staining. NK cell cytotoxicity was additionally measured by a chromium 
release assay (60): target tumor cells were labeled with $150 \mu \mathrm{Ci}{ }^{51} \mathrm{Cr}$ per $1 \times 10^{6}$ cells for 2 hours. Activated, purified, and nonpurified NK cells were prepared as described above and were then added at variable effector/target cell ratios from 1:1 to 50:1. Percentage of lysis was determined after 4 hours of culture in the presence of anti-CD137 mAb (BMS-663513, $10 \mu \mathrm{g} / \mathrm{ml})$ alone, cetuximab $(10 \mu \mathrm{g} / \mathrm{ml})$ alone, or cetuximab plus antiCD137 mAbs (both at $10 \mu \mathrm{g} / \mathrm{ml}$ ). All assays were performed in triplicate with 3 independent NK cell samples.

In vitro DC functional assays. Autologous iDCs were prepared as previously described (52). iDC populations were checked for CD1a and CD80 expression and lack of CD14 expression. Activated and purified NK cells were prepared as described above. Activation of NK cells was confirmed by flow cytometry ( $>50 \%$ expression of CD137). The activated NK cells were then cultured with autologous iDCs and EGFR-expressing cancer cell lines (SCC6, PC1, or SCC4) at a ratio of 1:1:1 for 18 hours in medium alone or with anti-CD137 mAb (BMS-663513, $10 \mu \mathrm{g} / \mathrm{ml}$ ) alone, cetuximab $(10 \mu \mathrm{g} / \mathrm{ml})$ alone, or cetuximab plus anti-CD137 mAbs (both at $10 \mu \mathrm{g} / \mathrm{ml}$ ); supernatant was harvested and analyzed by ELISA for IFN- $\gamma$, IL-12 p40, and TNF- $\alpha$ (human IFN- $\gamma$, IL-12 p 40 , and TNR- $\alpha$ BD OptEIA ELISA Sets and BD OptEIA Reagent Set B; BD Pharmingen). All assays were performed in triplicate with 3 independent NK:iDC cell samples.

Tumor transplantation and antibody therapy. SCC6 cancer cells were implanted s.c. into 5- to 6-week-old female athymic $n u / n u$ mice at a dose of $1 \times 10^{6}$ cells in $50 \mu \mathrm{l}$ of PBS (BD Biosciences). mAb were administered by i.p. injections. Cetuximab was given i.p. at $200 \mu \mathrm{g}$ per injection, and anti-CD137 mAb was given i.p. at $150 \mu \mathrm{g}$ per injection at various times after tumor inoculation. To determine the efficacy against KRAS-WT and mutant tumors, $n u / n u$ mice were inoculated with $1 \times 10^{6} \mathrm{~T} 84$ (KRAS-WT) tumor cells or $1 \times 10^{6}$ HCT116 (KRAS G13D mutant) tumor cells s.c. on the left flank. To determine the immune cell subsets necessary for efficacy, $\mathrm{BALB} / \mathrm{c}$ mice were inoculated with $1 \times 10^{6} \mathrm{TUBO}-\mathrm{EGFR}$ tumor cells s.c. on the left flank. Cetuximab and anti-CD137 mAb were administered after tumor inoculation as above. To characterize the adaptive immune response following combination therapy, BALB/c mice were inoculated with $1 \times 10^{6}$ TUBO-EGFR tumor cells s.c. on the left flank and $1 \times 10^{6}$ TUBO-EGFR tumor cells s.c. on the right flank. Cetuximab and anti-CD137 mAb were administered after tumor inoculation as above. Tumor growth was measured by caliper twice a week and expressed as the product of length by width in square centimeters. Mice were sacrificed when tumor size reached $4 \mathrm{~cm}^{2}$ or when tumor sites ulcerated. All in vivo models were piloted with 5 mice per group and repeated with 10 mice per group, except in the 2-tumor model, in which 15 mice were inoculated and 5 per group sacrificed on day 24 for tumor-infiltrating lymphocyte analysis and peripheral blood collection for assessment of $\mathrm{CD}^{+} \mathrm{T}$ cell function ex vivo.

Adoptive immunity transfer experiments (Winn assay). BALB/c mice were inoculated with $1 \times 10^{6}$ TUBO-EGFR tumor cells s.c. on the left flank and treated with either cetuximab monotherapy on days $14,21,28$, and 35 or sequential cetuximab and anti-CD137 mAb combination with cetuximab dosed as in the monotherapy group and anti-CD137 $\mathrm{mAb}$ on the subsequent day. All mice were sacrificed 50 days after being inoculated with tumor cells. Total or CD8-selected suspensions were prepared by CD8 $\mathrm{a}^{+} \mathrm{T}$ cell Isolation Kit (Miltenyi Biotec $\mathrm{GmbH}$ ) and purification assessed by flow cytometric analysis and confirmed as more than $95 \%$. Total or in vitro-purified CD8 ${ }^{+}$ splenocytes were admixed with TUBO or TUBO-EGFR tumor cells (100:1 ratio) and coinjected into naive $\mathrm{BALB} / \mathrm{c}$ mice. The results were expressed as percentage of animals that were tumor-free over time.

Detection of CD137-expressing cells in vivo, tumor-infiltrating immune cells in vivo, and IFN- $\gamma$ production assay ex vivo. Blood was collected from the tail vein, anticoagulated with $2 \mathrm{mM}$ EDTA in PBS, then diluted 1:1 with 2\% Dextran T500 (Pharmacosmos) in PBS and incubated at $37^{\circ} \mathrm{C}$ for 45 minutes to precipitate red cells. Leukocyte-containing supernatant was removed and centrifuged, and the remaining red cells were lysed with ACK Lysis Buffer (Quality Biological). Splenocytes and subcutaneous tumors from 3 mice per treatment group were resected 24,72 , or 168 hours following cetuximab treatment or isotype control in tumor-bearing mice and mechanically digested into a single-cell suspension. Cells were stained with mAbs to evaluate $\mathrm{CD} 137$ expression on $\mathrm{NK}$ cells by $\mathrm{CD} 3^{-} \mathrm{DX} 5^{+}$expression by flow cytometry. Subcutaneous TUBO and TUBO-EGFR tumors from 5 mice per treatment group were resected on day 24 after tumor inoculation and analyzed for NK cell, T cell, and DC infiltration by flow cytometry of a single-cell suspension following mechanical digestion. The tumor-infiltrating DC population was marked by $\mathrm{CD} 11 \mathrm{c}^{+}$and high surface expression of MHC class I and class II proteins with expression of costimulatory molecules CD80 (B7.1) and CD86 (B7.2). PBMCs collected on day 24 after tumor inoculation from 5 mice per group in the 2 tumor model were purified as described and then cocultured with medium, $1 \times 10^{6}$ irradiated A20, TUBO, or TUBO-EGFR cells for 24 hours with $0.5 \mathrm{mg}$ anti-mouse $\mathrm{CD} 28 \mathrm{mAb}$ (BD Biosciences - Pharmingen) and in the presence of monen$\sin$ (Golgistop; BD Biosciences) for the last 5 hours at $37^{\circ} \mathrm{C}$ and $5 \% \mathrm{CO}_{2}$. Intracellular IFN- $\gamma$ expression was assessed with BD Cytofix/Cytoperm Plus Kit per instructions.

Depletion of $\mathrm{CD}^{+}$and $\mathrm{CD}^{+} \mathrm{T}$ cells, NK cells, and macrophages. Anti-CD4 $\mathrm{mAb}$, anti-CD8 mAb, anti-asialo GM1 mAb (Wako Chemicals), and liposomal clodronate (Weissman laboratory, Stanford University) were used to deplete $\mathrm{CD}^{+}{ }^{+} \mathrm{T}$ cell, $\mathrm{CD} 8^{+} \mathrm{T}$ cell, $\mathrm{NK}$ cell, and macrophage activity in vivo, respectively. Ascitic fluid was harvested from SCID mice bearing hybridomas GK1.5- and GK2.43-producing anti-CD4 (rat IgG2b) and anti-CD8 (rat IgG2b) mAbs. The ascites were diluted in sterile PBS. Depleting anti$\mathrm{CD} 4 \mathrm{mAb}$, anti-CD8 $\mathrm{mAb}$, and anti-asialo GM1 $\mathrm{mAb}$ were injected i.p. on day -1 and day 0 of tumor inoculation, and every 5 days thereafter until day 45 at a dose of $500 \mu \mathrm{g}$ per injection of anti-CD4 mAb or anti-CD8 $\mathrm{mAb}$ and $50 \mu \mathrm{l}$ per injection of anti-asialo GM1 $\mathrm{mAb}$. Liposomal clodronate was injected retroorbitally on day -2 at a dose of $200 \mu$ and on day 0 of tumor inoculation, followed by every 4 days until day 44 at a dose of $100 \mu \mathrm{l}$ per injection. The depletion conditions were validated by flow cytometry of blood showing more than $95 \%$ depletion of each cell subset.

Antibodies for flow cytometry. mAbs to human antigens were used for staining of human PBMCs including the following: CD16 FITC, CD4 FITC, CD8 FITC, CD137 ligand PE, CD56 PE, CD3 PerCP, anti-EGFR APC, CD137 APC, APC-H7, FITC-annexin V, and 7-AAD (all from BD). HIVtetramer PE and EGFR-tetramer (YLN) PE were provided by the NIH Tetramer Core Facility (Atlanta, Georgia, USA) as well as Mark Davis (Stanford University). The following mAbs to mouse antigens were used: CD8 FITC (BD), CD4 FITC (BD), CD137 ligand PE (eBioscience), CD137 PE (eBioscience), CD3 PerCP (BD), DX5 APC (eBioscience), CD11c-PE (BD), MHC I APC (BD), MHC II APC (BD), B7.1 APC (BD), B7.2 FITC (BD), and F4/80 APC (eBioscience). Stained cells were collected on a FACSCalibur or a LSRII 3-laser cytometer (BD), and data were analyzed using Cytobank (http://www.cytobank.org) (61).

Analysis of Fc $\gamma$ RIIIa polymorphisms. Genomic DNA was prepared from peripheral blood mononuclear cells using a DNA extraction kit (QIAGEN). Genotyping of FcyRIIIa 158 V/F polymorphism was performed by a polymerase chain reaction followed by allele-specific restriction enzyme digestion (62). All genotyping of Fc $\gamma$ RIIIa polymorphism was confirmed by direct sequencing of the region of interest.

Statistics. Prism software (GraphPad) was used to analyze tumor growth and comparison of cell populations to determine statistical significance of differences (data presented as mean \pm SEM) between groups by applying a 2-tailed, unpaired Student's $t$ test or 2-way ANOVA with Bonferroni's correction for multiple comparisons. Kaplan-Meier plots were used to analyze 
survival. Comparisons of survival curves were made using the log-rank test. Correlation coefficients were calculated for comparison of percentage of $\mathrm{EGFR}^{+} \mathrm{CD}^{+} \mathrm{T}$ cells and percentage of $\mathrm{CD} 137^{+} \mathrm{NK}$ cells (data presented as mean $\pm \mathrm{SEM}$ ). $P<0.05$ was considered significant. For tumor burdens, comparisons of means were done by ANOVA.

Study approval. Human peripheral blood and primary breast tumor samples were obtained under a reviewed protocol approved by Stanford University's Administrative Panels on Human Subjects (NCT01114256) and following informed consent from patients in accordance with the Declaration of Helsinki. All animal experiments were approved by the Stanford Administrative Panel on Laboratory Animal Care and conducted in accordance with NIH guidelines.

\section{Acknowledgments}

This work was supported by the California Breast Cancer Research Program, EUREKA (R01 CA153248-01), the Reliable Cancer Therapy Fund, and Nadia's Gift Foundation at the Stanford Cancer Center. M.J. Goldstein is supported by the How- ard Hughes Medical Institute Research Training Fellowship. R. Houot is supported by fellowships from the Foundation de France and the Association pour la Recherche sur le Cancer. H.E. Kohrt is a Damon Runyon Clinical Investigator and is supported by fellowships from the American Society of Hematology and the American Society of Clinical Oncology and Department of Defense Breast Cancer postdoctoral fellowship award (26687). $\mathrm{K}$. Weiskopf is supported by the Stanford University Medical Scientist Training Program. R. Levy is an American Cancer Society Clinical Research Professor.

Received for publication September 3, 2013, and accepted in revised form March 6, 2014.

Address correspondence to: Holbrook Kohrt, Division of Oncology, 269 Campus Drive, CCSR 1105, Stanford University Medical Center, Stanford, California 94305-5151, USA. Phone: 650.725.6452; Fax: 650.736.1454; E-mail: Kohrt@stanford.edu.
1. American Cancer Society. Cancer Facts and Figures 2013. Atlanta, Georgia, USA: American Cancer Society; 2013.

2. Lee CM, Tannock IF. The distribution of the therapeutic monoclonal antibodies cetuximab and trastuzumab within solid tumors. BMC Cancer. 2010;10:255.

3. De Roock W, De Vriendt V, Normanno N, Ciardiello F, Tejpar S. KRAS, BRAF, PIK3CA, and PTEN mutations: implications for targeted therapies in metastatic colorectal cancer. Lancet Oncol. 2011; 12(6):594-603.

4. Baselga J, et al. Phase II multicenter study of the antiepidermal growth factor receptor monoclonal antibody cetuximab in combination with platinum-based chemotherapy in patients with platinum-refractory metastatic and/or recurrent squamous cell carcinoma of the head and neck. J Clin Oncol. 2005;23(24):5568-5577.

5 . Vermorken JB, et al. Open-label, uncontrolled, multicenter phase II study to evaluate the efficacy and toxicity of cetuximab as a single agent in patients with recurrent and/or metastatic squamous cell carcinoma of the head and neck who failed to respond to platinum-based therapy. J Clin Oncol. 2007; 25(16):2171-2177.

6. Liu L, Cao Y, Tan A, Liao C, Gao F. Cetuximabbased therapy versus non-cetuximab therapy for advanced cancer: a meta-analysis of 17 randomized controlled trials. Cancer Chemother Pharmacol. 2010; 65(5):849-861.

7. Li X, Lu Y, Liang K, Pan T, Mendelsohn J, Fan Z. Requirement of hypoxia-inducible factor-1alpha down-regulation in mediating the antitumor activity of the anti-epidermal growth factor receptor monoclonal antibody cetuximab. Mol Cancer Ther. 2008;7(5):1207-1217.

8. Huang SM, Li J, Harari PM. Molecular inhibition of angiogenesis and metastatic potential in human squamous cell carcinomas after epidermal growth factor receptor blockade. Mol Cancer Ther. 2002; 1(7):507-514.

9. Clynes RA, Towers TL, Presta LG, Ravetch JV. Inhibitory $F_{C}$ receptors modulate in vivo cytoxicity against tumor targets. Nat Med. 2000;6(4):443-446.

10. Kurai J, et al. Antibody-dependent cellular cytotoxicity mediated by cetuximab against lung cancer cell lines. Clin Cancer Res. 2007;13(5):1552-1561.

11. Steiner $\mathrm{P}$, et al. Tumor growth inhibition with cetuximab and chemotherapy in non-small cell lung cancer xenografts expressing wild-type and mutated epidermal growth factor receptor. Clin Cancer Res. 2007;13(5):1540-1551.

12. Raben $\mathrm{D}$, et al. The effects of cetuximab alone and in combination with radiation and/or chemotherapy in lung cancer. Clin Cancer Res. 2005;11(2 pt 1):795-805

13. Wilson NS, et al. An Fc $\gamma$ receptor-dependent mechanism drives antibody-mediated targetreceptor signaling in cancer cells. Cancer Cell. 2011; 19(1):101-113.

14. Marechal R, et al. Putative contribution of CD56 positive cells in cetuximab treatment efficacy in first-line metastatic colorectal cancer patients. BMC Cancer. 2010;10:340.

15. Lopez-Albaitero A, et al. Role of polymorphic Fc $\gamma$ receptor IIIa and EGFR expression level in cetuximab mediated, NK cell dependent in vitro cytotoxicity of head and neck squamous cell carcinoma cells. Cancer Immunol Immunother. 2009;58(11):1853-1864.

16. Bibeau F, et al. Impact of Fc\{gamma\}RIIaFc $\{$ gamma\}RIIIa polymorphisms and KRAS mutations on the clinical outcome of patients with metastatic colorectal cancer treated with cetuximab plus irinotecan. J Clin Oncol. 2009;27(7):1122-1129.

17. Ferris RL, Jaffee EM, Ferrone S. Tumor antigen-targeted, monoclonal antibody-based immunotherapy: clinical response, cellular immunity, and immunoescape. J Clin Oncol. 2010;28(28):4390-4399.

18. Qiu LX, et al. Predictive and prognostic value of KRAS mutations in metastatic colorectal cancer patients treated with cetuximab: a meta-analysis of 22 studies. Eur J Cancer. 2010;46(15):2781-2787.

19. Rodriguez J, et al. Fc $\gamma$ receptor polymorphisms as predictive markers of Cetuximab efficacy in epidermal growth factor receptor downstream-mutated metastatic colorectal cancer. Eur J Cancer. 2012; 48(12):1774-1780.

20. Lee SC, Srivastava RM, Lopez-Albaitero A, Ferrone S, Ferris RL. Natural killer (NK): dendritic cell (DC) cross talk induced by therapeutic monoclonal antibody triggers tumor antigen-specific $\mathrm{T}$ cell immunity. Immunol Res. 2011;50(2-3):248-254.

21. Lin W, et al. Fc-dependent expression of CD137 on human NK cells: insights into "agonistic" effects of anti-CD137 monoclonal antibodies. Blood. 2008; 112(3):699-707.

22. Yang X, Zhang X, Mortenson ED, RadkevichBrown O, Wang Y, Fu YX. Cetuximab-mediated tumor regression depends on innate and adaptive immune responses. Mol Ther. 2013;21(1):91-100.

23. Schuler PJ, et al. EGFR-specific T cell frequencies correlate with EGFR expression in head and neck squamous cell carcinoma. J Transl Med. 2011;9:168.

24. Srivastava RM, et al. Cetuximab-activated natural killer and dendritic cells collaborate to trigger tumor antigen-specific T-cell immunity in head and neck cancer patients. Clin Cancer Res. 2013; 19(7):1858-1872.
25. Mendelsohn J, Baselga J. Status of epidermal growth factor receptor antagonists in the biology and treatment of cancer. J Clin Oncol. 2003; 21(14):2787-2799.

26. Dassonville O, et al. Expression of epidermal growth factor receptor and survival in upper aerodigestive tract cancer. J Clin Oncol. 1993; 11(10):1873-1878.

27. Kohrt HE, et al. CD137 stimulation enhances the antilymphoma activity of anti-CD20 antibodies. Blood. 2011;117(8):2423-2432.

28. Kohrt HE, et al. Stimulation of natural killer cells with a CD137-specific antibody enhances trastuzumab efficacy in xenotransplant models of breast cancer. J Clin Invest. 2012;122(3):1066-1075.

29. Melero I, Hervas-Stubbs S, Glennie M, Pardoll DM, Chen L. Immunostimulatory monoclonal antibodies for cancer therapy. Nat Rev Cancer. 2007;7(2):95-106.

30. Wilcox RA, et al. Cutting edge: Expression of functional CD137 receptor by dendritic cells. J Immunol. 2002;168(9):4262-4267.

31. Narazaki H, Zhu Y, Luo L, Zhu G, Chen L. CD137 agonist antibody prevents cancer recurrence: contribution of CD137 on both hematopoietic and nonhematopoietic cells. Blood. 2010;115(10):1941-1948.

32. Baessler T, et al. CD137 ligand mediates opposite effects in human and mouse NK cells and impairs NK cell reactivity against human acute myeloid leukemia cells. Blood. 2010;115(15):3058-3069.

33. Melero I, Johnston JV, Shufford WW, Mittler RS, Chen L. NK1.1 cells express 4-1BB (CDw137) costimulatory molecule and are required for tumor immunity elicited by anti-4-1BB monoclonal antibodies. Cell Immunol. 1998;190(2):167-172.

34. Wilcox RA, Tamada K, Strome SE, Chen L. Signaling through NK cell-associated CD137 promotes both helper function for $\mathrm{CD}^{+}$cytolytic T cells and responsiveness to IL-2 but not cytolytic activity. JImmunol. 2002;169(8):4230-4236.

35. Maniar A, et al. Human \{gamma\}\{delta\} T lymphocytes induce robust NK cell mediated antitumor cytotoxicity through CD137 engagement. Blood. 2010;116(10):1726-1733

36. Gong W, et al. Immobilized MHC class I chainrelated protein A synergizes with IL-15 and soluble 4-1BB ligand to expand NK cells with high cytotoxicity ex vivo. Cell Mol Immunol. 2010; 7(6):477-484

37. Kohrt HE, et al. CD137 stimulation enhances the anti-lymphoma activity of anti-CD20 antibodies. Blood. 2010;117(8):2423-2432.

38. Cooper MA, Fehniger TA, Caligiuri MA. The biology of human natural killer-cell subsets. Trends Immunol. 2001;22(11):633-640. 
39. Bowles JA, Weiner GJ. CD16 polymorphisms and NK activation induced by monoclonal antibody-coated target cells. J Immunol Methods. 2005;304(1-2):88-99.

40. Juelke $\mathrm{K}$, et al. CD62L expression identifies a unique subset of polyfunctional CD56dim NK cells. Blood. 2010;116(8):1299-1307.

41. Mani A, et al. A phase II trial of trastuzumab in combination with low-dose interleukin-2 (IL-2) in patients (PTS) with metastatic breast cancer (MBC) who have previously failed trastuzumab. Breast Cancer Res Treat. 2009;117(1):83-89.

42. Acquavella N, et al. Toxicity and activity of a twice daily high-dose bolus interleukin 2 regimen in patients with metastatic melanoma and metastatic renal cell cancer. J Immunother. 2008;31(6):569-576.

43. Gluck WL, et al. Phase I studies of interleukin (IL)-2 and rituximab in B-cell non-hodgkin's lymphoma: IL-2 mediated natural killer cell expansion correlations with clinical response. Clin Cancer Res. 2004; 10(7):2253-2264.

44. Ansell SM, et al. Phase 1 study of interleukin-12 in combination with rituximab in patients with B-cell non-Hodgkin lymphoma. Blood. 2002;99(1):67-74

45. Houot R, et al. Therapeutic effect of CD137 immunomodulation in lymphoma and its enhancement by Treg depletion. Blood. 2009;114(16):3431-3438.

46. Kohrt HE, et al. CD137 stimulation enhances the anti-lymphoma activity of anti-CD20 antibodies. Blood. 2011;117(8):2423-2432.

47. Genovesi EV, Pettey CL, Collins JJ. Use of adoptive transfer and Winn assay procedures in the further analysis of antiviral acquired immunity in mice protected against Friend leukemia virus-induced disease by passive serum therapy. Cancer Res. 1984; 44(4):1489-1498.

48. Jiang D, Schwarz H. Regulation of granulocyte and macrophage populations of murine bone marrow cells by G-CSF and CD137 protein. PLoS One. 2010; 5(12): 15565

49. Zhang $X$, et al. CD137 promotes proliferation and survival of human B cells. J Immunol. 2010; 184(2):787-795.

50. Murillo O, et al. In vivo depletion of DC impairs the anti-tumor effect of agonistic anti-CD137 mAb. Eur IImmunol. 2009;39(9):2424-2436.

51. Sharma RK, et al. 4-1BB ligand as an effective multifunctional immunomodulator and antigen delivery vehicle for the development of therapeutic cancer vaccines. Cancer Res. 2010;70(10):3945-3954.

52. Piccioli D, Sbrana S, Melandri E, Valiante NM. Contact-dependent stimulation and inhibition of dendritic cells by natural killer cells. J Exp Med. 2002; 195(3):335-341.

53. Wehner R, Dietze K, Bachmann M, Schmitz M. The bidirectional crosstalk between human dendritic cells and natural killer cells. J Innate Immun. 2011; 3(3):258-263

54. Ollion V, et al. Tumor cell-derived Ag cross-presentation in human DC induced by NK cells: mechanisms and specialization of the BDCA- $3^{+}$DC subset. Presented at: 15th International Congress of Immunology; August 22-27, 2013; Milan, Italy. Abstract P1.08.18.

55. Gerosa F, Baldani-Guerra B, Nisii C, Marchesini V, Carra G, Trinchieri G. Reciprocal activating interaction between natural killer cells and dendritic cells. J Exp Med. 2002;195(3):327-333.

56. Mailliard RB, et al. Dendritic cells mediate NK cell help for Th1 and CTL responses: two-signal requirement for the induction of NK cell helper function. J Immunol. 2003;171(5):2366-2373.

57. Agaugue S, Marcenaro E, Ferranti B, Moretta L, Moretta A. Human natural killer cells exposed to IL-2, IL-12, IL-18, or IL-4 differently modulate priming of naive $\mathrm{T}$ cells by monocyte-derived dendritic cells. Blood. 2008;112(5):1776-1783.

58. Matsuo T, Nishizuka SS, Ishida K, Iwaya T, Ikeda M, Wakabayashi G. Analysis of the anti-tumor effect of cetuximab using protein kinetics and mouse xenograft models. BMC Res Notes. 2011;4:140.

59. Wilcox RA, et al. Provision of antigen and CD137 signaling breaks immunological ignorance, promoting regression of poorly immunogenic tumors. J Clin Invest. 2002;109(5):651-659.

60. Kim GG, Donnenberg VS, Donnenberg AD, Gooding W, Whiteside TL. A novel multiparametric flow cytometry-based cytotoxicity assay simultaneously immunophenotypes effector cells: comparisons to a 4 h 51Cr-release assay. J Immunol Methods. 2007; 325(1-2):51-66

61. Kotecha N, et al. Single-cell profiling identifies aberrant STAT5 activation in myeloid malignancies with specific clinical and biologic correlates. Cancer Cell. 2008;14(4):335-343.

62. Koene HR, Kleijer M, Algra J, Roos D, von dem Borne AE, de Haas M. FcyRIIIa-158V/F polymorphism influences the binding of IgG by natural killer cell FcyRIIIa, independently of the FcyRIIIa48L/R/H phenotype. Blood. 1997;90(3):1109-1114. 